\title{
Correlation between optical and $\gamma$-ray flux variations in bright flat spectrum radio quasars
}

\author{
Bhoomika $^{1 \star}$, C. S. Stalin ${ }^{1}$, S. Sahayanathan ${ }^{2}$ \\ ${ }^{1}$ Indian Institute of Astrophysics, Block II, Koramangala, Bangalore 560034, India \\ ${ }^{2}$ Astrophysical Sciences Division, Bhabha Atomic Research Centre, Mumbai, India
}

Last updated 2015 May 22; in original form 2013 September 5

\begin{abstract}
Blazars are known to show flux variations over a range of energies from low energy radio to high energy $\gamma$-rays. Cross-correlation analysis of the optical and $\gamma$-ray light curves in blazars shows that flux variations are generally correlated in both bands, however, there are exceptions. We explored this optical-GeV connection in four flat spectrum radio quasars (FSRQs) by a systematic investigation of their long term optical and $\gamma$-ray light curves. On analysis of the four sources, namely 3C 273, 3C 279, PKS 1510-089 and CTA 102 we noticed different behaviours between the optical and GeV flux variations. We found instances when (i) the optical and $\mathrm{GeV}$ flux variations are closely correlated (ii) there are optical flares without $\gamma$-ray counterparts and (iii) $\gamma$-ray flares without optical counterparts. To understand these diverse behaviours, we carried out broad band spectral energy distribution (SED) modelling of the sources at different epochs using a one-zone leptonic emission model. The optical-UV emission is found to be dominated by emission from the accretion disk in the sources PKS 1510-089, CTA 102 and 3C 273, while in 3C 279, the synchrotron radiation from the jet dominates the optical-UV emission. Our SED analysis indicates that (i) correlated optical and $\gamma$-ray flux variations are caused by changes in the bulk Lorentz factor $(\Gamma$ ), (ii) $\gamma$-ray flares without optical counterparts are due to increase in $\Gamma$ and/or the electron energy density and (iii) an optical flare without $\gamma$-ray counterpart is due to increase in the magnetic field strength.
\end{abstract}

Key words: galaxies: active - galaxies: nuclei - galaxies: jets - $\gamma$-rays:galaxies

\section{INTRODUCTION}

Blazars, among the most luminous objects $\left(10^{42}-10^{48} \mathrm{erg} \mathrm{s}^{-1}\right)$ in the Universe, are a class of active galactic nuclei (AGN) believed to be powered by accretion of matter onto super massive black holes with masses greater than $\sim 10^{6} \mathrm{M}_{\odot}$ situated at the centers of galaxies (Lynden-Bell 1969; Shakura \& Sunyaev 1973). These objects have relativistic jets oriented close to the line of sight (within a few degrees) to the observer and their radiation output is dominated by non-thermal emission processes (Antonucci 1993; Urry \& Padovani 1995). They display rapid and large amplitude flux variability over the entire accessible wavelength band on a range of time scales from minutes to years (Wagner \& Witzel 1995; Ulrich et al. 1997). In addition to flux variations, blazars also show high polarization (Kinman et al. 1966; Angel \& Stockman 1980) and polarization variability (Andruchow et al. 2005; Abdo et al. 2010a; Rakshit et al. 2017; Rani et al. 2018; Pasierb et al. 2020). Blazars are divided into flat spectrum radio quasars (FSRQs) and BL Lac objects (BL Lacs) with FSRQs having strong emission lines in their optical/infra-red (IR) spectra while BL Lacs have either featureless spectra or spectra with weak emission lines, with equivalent widths $<5 \AA$. A physical distinction between FSRQs and BL Lacs has been put forward by Ghisellini et al. (2011) with FSRQs having the ratio of the luminosity of the

\footnotetext{
^ E-mail: bhoomika@iiap.res.in
}

broad line region $\left(L_{B L R}\right)$ to the Eddington luminosity $\left(L_{E d d}\right)>5$ $\times 10^{-5}$. A two hump structure is evident in the broad band spectral energy distribution (SED) of blazars, the low energy component peaking in the IR-X-ray band and the high energy component peaking in the MeV - GeV band (Fossati et al. 1998; Mao et al. 2016). In the leptonic scenario, the low energy component is attributed to synchrotron emission process by the relativistic electrons in the jet, while the high energy component is attributed to inverse Compton (IC) process (Abdo et al. 2010d). The seed photons for IC emission could be the synchrotron photons from the jet (synchroton self Compton SSC; Konigl 1981; Marscher \& Gear 1985; Ghisellini \& Maraschi 1989) as well as photons exterior to the jet (external compton EC; Begelman et al. 1987). These external photons can be from the accretion disk (Dermer \& Schlickeiser 1993; Boettcher et al. 1997), the BLR (Ghisellini \& Madau 1996; Sikora et al. 1994) and the torus (Błażejowski et al. 2000; Ghisellini \& Tavecchio 2008).

The observed broad band SED of blazars are generally explained satisfactorily by leptonic models, however, there are exceptions, wherein the observed SED is interpreted by hadronic (Mücke et al. 2003; Böttcher et al. 2013; Rajput et al. 2019) or lepto-hadronic models (Diltz \& Böttcher 2016; Paliya et al. 2016). In the hadronic models of blazars, the high energy emission is due to synchrotron emission from protons that are accelerated to relativistic energies (Mücke et al. 2003; Aharonian 2000) or from pair cascades initiated by proton-proton or proton-photon interactions (Mannheim 1993). 
Table 1. Details of the objects analysed in this work. The mean $\gamma$-ray flux in the $100 \mathrm{MeV}-300 \mathrm{GeV}$ band is in units of $10^{-7} \mathrm{ph} \mathrm{cm}^{-2} s^{-1}$ and $\Gamma_{p}$ is the $\gamma$-ray photon index in the $100 \mathrm{MeV}-300 \mathrm{GeV}$ band. The values of $\Gamma_{p}$ are from (Ackermann et al. 2015).

\begin{tabular}{lcccccr}
\hline Name & 4FGL name & $\alpha_{2000}$ & $\delta_{2000}$ & $z$ & $\Gamma_{p}$ & $\gamma$-ray flux \\
\hline PKS 1510-089 & 4FGL J1512.8-0906 & $15: 12: 50.53$ & $-09: 05: 59.83$ & 0.360 & 2.364 & 9.13 \\
3C 273 & 4FGL J1229.1+0202 & $12: 29: 06.70$ & $+02: 03: 08.60$ & 0.158 & 2.661 & 6.73 \\
3C 279 & 4FGL J1256.1-0547 & $12: 56: 11.17$ & $-05: 47: 21.52$ & 0.536 & 2.343 & 8.79 \\
CTA 102 & 4FGL J2232.5+1143 & $22: 32: 36.41$ & $+11: 43: 50.90$ & 1.037 & 2.520 & 13.70 \\
\hline
\end{tabular}

Recent observations indicate that a single model might be inadequate to explain the SED of a source at all times. For example, in the broad band SED analysis of 3C 279 at various epochs, it has been found that leptonic model explains the SED during the March - April 2014 flare (Paliya et al. 2015), while the SED during the flare in December 2013 is better fit by lepto-hardronic models (Paliya et al. 2016). TXS 0506+056 is the first blazar associated with the detection of neutrinos by the IceCube neutrino observatory on 22 September 2017 and this was coincident in direction and time with a $\gamma$-ray flare from TXS 0506+056. This gives observational evidence of hadronic emission in blazars (IceCube Collaboration et al. 2018). Also, recently another blazar has been found to be spatially coincident with the IceCube neutrino event IC-200107A (Paliya et al. 2020). Thus, it is very clear that we do not yet fully understand the emission processes that contribute to the high energy emission in blazars.

Broad band SED modelling of blazars is often used to constrain the hadronic v/s leptonic scenario for the production of high energy $\gamma$-ray emission in them. An alternative to this SED based approach is the one based on carrying out a comparative analysis of the flux variations in the optical and $\gamma$-ray bands. In the leptonic scenario, as the relativistic electrons in the jets of blazars are responsible for both the optical and $\gamma$-ray emission a close correlation is expected between the optical and $\gamma$-ray flux variations (Böttcher 2007). Alternatively, in the hadronic model of emission from blazars, though the optical emission is dominated by electron synchrotron, the $\gamma$-ray emission could be from proton synchrotron, and therefore, a correlation between the optical and $\gamma$-ray flux variations may not be expected (Mücke \& Protheroe 2001). Thus, by a systematic investigation of the correlation between the optical and $\gamma$-ray flux variations in a sample of blazars, it would be possible to constrain the leptonic v/s hardonic emission from blazar jets. An alternative to SED modelling and optical - $\gamma$-ray studies to distinguish between the leptonic and hadronic scenarios in the high energy emission from FSRQs is through their X-ray polarization. According to Zhang \& Böttcher (2013) X-ray polarization in blazars will be different in these two scenarios. Xray polarimetric observations in the future from the Imaging X-ray Polarimetry Explorer (IXPE; Weisskopf et al. 2016) will be able to constrain the origin of high energy emission in blazars.

The launch of the Fermi Gamma-ray Space Telescope (hereinafter Fermi; Atwood et al. 2009) in the year 2008 has enabled investigation of the long term $\gamma$-ray flux variability characteristics of blazars (Rajput et al. 2020) that dominate the extragalactic $\gamma$-ray sky. Prior to Fermi, the availability of long term $\gamma$-ray light curves of blazars were limited. However, today we know about 3000 blazars that are detected by Fermi (The Fermi-LAT collaboration 2019) and most of them have $\gamma$-ray light curves spanning more than 10 years ${ }^{1}$ suitable for long term $\gamma$-ray variability studies. In support of Fermi, ground based monitoring observations in the optical and IR are being carried out by the Small and Moderate Aperture Research Telescope Sys- tem (SMARTS ${ }^{2}$; Bonning et al. 2009) and the Steward Observatory ${ }^{3}$ (Smith et al. 2009). These observations in the optical and IR bands serve as a valuable data set to study the correlations between the optical and $\gamma$-ray flux variations in blazars. Studies carried out on these lines have led to varied results. Few studies demonstrated that the $\gamma$-ray flares in blazars are correlated with optical flares with or without lag (Bonning et al. 2009; Chatterjee et al. 2012; Liao et al. 2014; Carnerero et al. 2015). However, studies of this kind carried out on more objects have found that the optical and $\gamma$-ray flux variations are not correlated all times and there are objects where $\gamma$-ray flares are detected without an optical counterpart (Vercellone et al. 2011; Dutka et al. 2013; MacDonald et al. 2015). Similarly, prominent optical flares with no corresponding $\gamma$-ray flares are also known in some objects (Chatterjee et al. 2013; Cohen et al. 2014; Rajput et al. 2019). Recently, Liodakis et al. (2019) looked for the presence/absence of correlated optical and $\gamma$-ray flux variations in a large sample of Fermi blazars. To further probe the prevalence of anomalous optical and $\gamma$-ray flux variability in blazars and understand their physical characteristics through broad band SED modelling, we carried out a systematic analysis of the $\gamma$-ray flux variability of blazars that have optical and IR monitoring data available in the archives. Here, we present our results on four FSRQs. Results on the BL Lacs analyzed as part of this investigation will be presented elsewhere. In section 2, we provide the details on the selection of the objects for this program. The data used in this work is explained in Section 3 followed by the analysis in Section 4. The results are presented in Section 5 followed by the summary in the final section.

\section{SAMPLE}

For this work, we first selected all sources that are classified as FSRQs in the third catalog of AGN detected by the large area telescope (LAT) onboard Fermi (3LAC; Ackermann et al. 2015). For the selected FSRQs we then looked into their one day binned $\gamma$-ray light curves given at the Fermi site ${ }^{4}$ and selected those sources that have at least one flare with the $\gamma$-ray flux exceeding $10^{-6}$ photons $\mathrm{cm}^{-2} \mathrm{~s}^{-1}$. This lead us to a sample of 84 sources. For those 84 sources, we looked at the archives of SMARTS for the availability of optical and IR data overlapping the duration of $\gamma$-ray light curves. For 40 out of the 84 sources we found data in SMARTS. Of these 40, three sources namely 3C 454.3, PKS 1510-089 and 3C 279 have the largest number of data points in the optical and IR bands with the total exceeding 500. To these three, we added two more sources namely CTA 102 and 3C 273 due to their high $\gamma$-ray activity states (Ciprini 2016; Bastieri 2009). Thus, our final sample for correlated optical - GeV studies consists of five sources. Of these five, results for one source 3C 454.3 is already published in Rajput et al. (2019). In this work we present our results on the analysis of the remaining four sources. The details of these

\footnotetext{
${ }^{1}$ https://fermi.gsfc.nasa.gov/ssc/data/access/lat/msl_lc/
}

\footnotetext{
2 http://www.astro.yale.edu/smarts/glast/home.php

3 http://james.as.arizona.edu/ psmith/Fermi/\#mark6

4 https://fermi.gsfc.nasa.gov/ssc/data/access/lat/msl_lc/
} 
Table 2. Details of the epochs considered for detailed light curve analysis, SED modelling and spectral analysis. The $\gamma$-ray fluxes in the $100 \mathrm{MeV}$ to $300 \mathrm{GeV}$ band are in units of $10^{-6} \mathrm{ph} \mathrm{cm}^{-2} \mathrm{~s}^{-1}$ and the optical fluxes in the V-band are in units of $10^{-11} \mathrm{erg} \mathrm{cm}^{-2} \mathrm{~s}^{-1}$

\begin{tabular}{|c|c|c|c|c|c|c|c|c|}
\hline \multirow[b]{2}{*}{ Name } & \multirow[b]{2}{*}{ ID } & \multicolumn{2}{|c|}{ MJD } & \multicolumn{2}{|c|}{ Calendar date } & \multicolumn{2}{|c|}{ Mean flux } & \multirow[b]{2}{*}{ Remark } \\
\hline & & Start & End & Start & End & $\gamma$ & Optical & \\
\hline \multirow[t]{6}{*}{ PKS $1510-089$} & $\mathrm{~A}$ & 54937 & 54957 & 16-04-2009 & 06-05-2009 & 2.97 & 1.07 & $\gamma$-ray flare with no optical flare \\
\hline & B & 54951 & 54971 & 30-04-2009 & 20-05-2009 & 2.26 & 1.94 & $\gamma$-ray flare and optical flare \\
\hline & $\mathrm{C}$ & 55757 & 55777 & $15-07-2011$ & 04-08-2011 & 1.10 & 0.66 & $\gamma$-ray flare with no optical flare \\
\hline & $\mathrm{D}$ & 56062 & 56162 & $15-05-2012$ & 23-08-2012 & 0.44 & 0.62 & Quiescent state \\
\hline & $\mathrm{E}$ & 57105 & 57125 & 24-03-2015 & $13-04-2015$ & 3.12 & 0.95 & $\gamma$-ray flare with no optical flare \\
\hline & $\mathrm{F}$ & 57157 & 57177 & $15-05-2015$ & 04-06-2015 & 3.17 & 2.30 & $\gamma$-ray flare and optical flare \\
\hline \multirow[t]{2}{*}{$3 \mathrm{C} 273$} & A & 55265 & 55285 & $10-03-2010$ & $30-03-2010$ & 1.53 & 16.9 & $\gamma$-ray flare with no optical flare \\
\hline & B & 56450 & 56550 & 07-06-2013 & $15-09-2013$ & 0.40 & 16.7 & Quiescent state \\
\hline \multirow[t]{4}{*}{$3 \mathrm{C} 279$} & A & 55290 & 55390 & $04-04-2010$ & $13-07-2010$ & 0.26 & 0.14 & Quiescent state \\
\hline & B & 56742 & 56762 & 26-03-2014 & $15-04-2014$ & 2.21 & 2.15 & $\gamma$-ray flare with no optical flare \\
\hline & $\mathrm{C}$ & 57178 & 57198 & 05-06-2015 & $25-06-2015$ & 3.94 & 1.42 & $\gamma$-ray flare with no optical flare \\
\hline & $\mathrm{D}$ & 57828 & 57848 & $16-03-2017$ & 05-04-2017 & 2.33 & 4.25 & optical flare but no $\gamma$-ray flare \\
\hline \multirow[t]{2}{*}{ CTA 102} & A & 55840 & 55940 & 06-10-2011 & $14-01-2012$ & 0.31 & 0.39 & Quiescent state \\
\hline & $\mathrm{B}$ & 57740 & 57750 & $18-12-2016$ & 28-12-2016 & 10.3 & 44.5 & $\gamma$-ray flare and optical flare \\
\hline
\end{tabular}

four sources are given in Table 1. A brief description about them are given below:

\subsection{PKS 1510-089}

It was identified as a quasar firstly by Bolton \& Ekers (1966) with a visual magnitude of 16.5 mag. It is one of the most variable FSRQs in the 3FGL catalog. Located at a redshift of $z=0.361$ (Tanner et al. 1996), it is powered by a black hole of mass $5.4 \times 10^{8} \mathrm{M}_{\odot}$ and accretes at the rate of $0.5 \mathrm{M}_{\odot} /$ year (Abdo et al. 2010e). It has been detected at very high energies by HESS (H. E. S. S. Collaboration et al. 2013) and MAGIC (Major Atmospheric Gamma-Ray Imaging Cherenkov; Aleksić et al. 2014). This source has been studied for multi-wavelength flux variability (Prince et al. 2017; Nalewajko 2013) as well as subjected to few SED modelling studies (Prince et al. 2019; Nalewajko et al. 2012). Considering radio observations with the VLBA coupled with optical long term monitoring data $\mathrm{Wu}$ et al. (2005) argued for the presence of a binary black hole in PKS 1510-089.

\section{$2.23 \mathrm{C} 273$}

3C 273, the first quasar discovered by Schmidt (1963) at a redshift $z=$ 0.158 has a large scale radio jet with a projected size of $57 \mathrm{kpc}$ (Harris $\&$ Krawczynski 2006). It was the first quasar that was discovered in the $\gamma$-ray band in the energy range of 50-500 MeV (Swanenburg et al. 1978). It was later detected by the Energetic Gamma- Ray Experiment Telescope (EGRET; Hartman et al. 1999) and then by Fermi. It has been studied for flux variations in the optical (Xiong et al. 2017) and also has been found to show dramatic variations in the $\gamma$-ray band from Fermi observations (Abdo et al. 2010c). The $\gamma$-ray outburst in 2009 was explained by a time dependent one zone synchrotron self-Compton model (Zheng et al. 2013).

\section{$2.33 \mathrm{C} 279$}

At a redshift of $z=0.536$ (Lynds et al. 1965), 3C 279 was among the blazars that were discovered as emitters of $\gamma$-rays by EGRET (Hartman et al. 1992). In the GeV-TeV range it was first detected by the ground based atmospheric Cherenkov experiment MAGIC (MAGIC Collaboration et al. 2008). It has been recently suggested that 3C 279 hosts a supermassive black hole binary at its center
(Qian et al. 2019). The source is found to show flux variations over a range of wavelengths such as radio (Pauliny-Toth \& Kellermann 1966), optical (Oke 1967) and $\gamma$-rays (Hartman et al. 1992). It has also been studied for correlated variations over different wavebands (Chatterjee et al. 2008). Fermi observations have revealed minute scale flare in this source with a shortest flux doubling time scale lesser than 5 minutes during the outburst in 2015 (Hayashida et al. 2017). In addition to flux variability studies, it has also been studied via broad band SED modelling during various activity states. The flares at different epochs of the source were explained by leptonic process (Paliya et al. 2015; Shah et al. 2019), lepto-hardonic process (Paliya et al. 2018) as well as hadronic processes (Petropoulou et al. 2017). These observations and subsequent modelling clearly indicate that the same emission mechanisms are not responsible for the high energy emission we receive from the source at all times.

\subsection{CTA 102}

This FSRQ at a redshift of $z=1.037$ (Schmidt 1965) is highly polarized (Moore \& Stockman 1981) and variable in the optical band (Maraschi et al. 1986). It was detected in the $\gamma$-ray band both by EGRET (Fichtel et al. 1994) and Fermi (Abdo et al. 2009). It has been studied for flux variations across different wavebands (Kaur \& Baliyan 2018) and minute like time scales of variability were detected in the optical (Osterman Meyer et al. 2009) and $\gamma$-ray bands (Shukla et al. 2018).

\section{REDUCTION OF MULTI-WAVELENGTH DATA}

Our aim in this work is to characterize the connection between optical and $\gamma$-ray flux variations in FSRQs. Therefore, primarily data in both the optical and $\gamma$-ray bands are needed. However, for broad band SED modelling, data from other wavelength regions are also required. Thus, for this work we used all the publicly available data in the IR, optical, UV, X-rays and $\gamma$-rays that span a period of 10 years between 08 August 2008 and 08 August 2018. Optical polarimetric data if available during the above period was also used. 


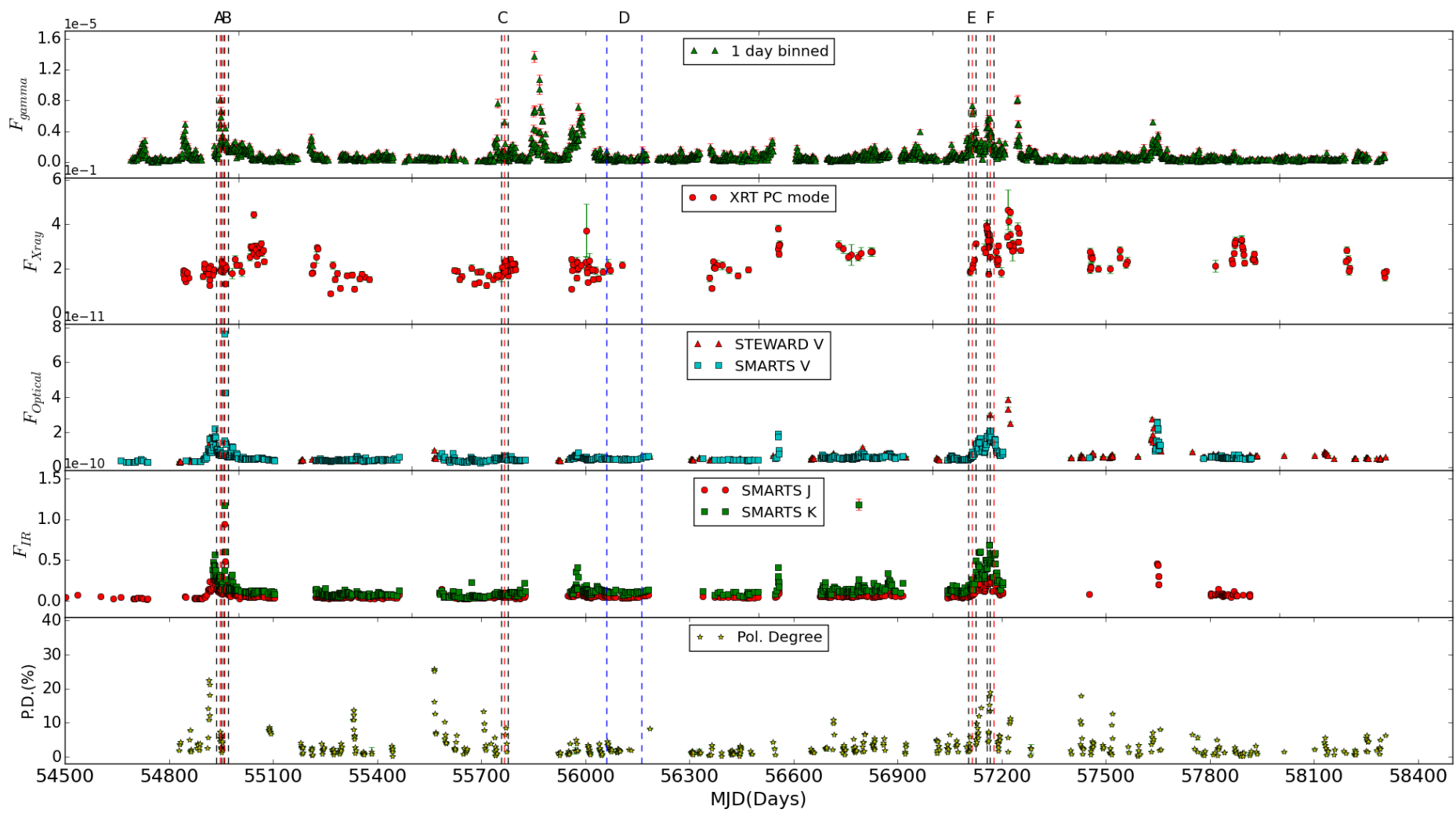

Figure 1. Light curves of the source PKS 1510-089. The top one is the one day binned $\gamma$-ray light curve (in units of $10^{-5} \mathrm{ph} \mathrm{cm}^{-2} \mathrm{~s}^{-1}$ ), the second panel from the top is the X-ray light curve (in units of counts/sec), the next two panels are the optical (in units of $10^{-11} \mathrm{erg} \mathrm{cm}^{-2} \mathrm{~s}^{-1}$ ) and the IR (in units of $10^{-10} \mathrm{erg}$ $\mathrm{cm}^{-2} \mathrm{~s}^{-1}$ ) light curves and the bottom panel is the optical V-band polarization. The peak of either the optical or $\gamma$-ray light curve is shown by red dotted lines, while the two black solid lines on either side of the red line correspond to a width of 10 days each. The two blue lines show the quiescent period of 100 days. For the $\gamma$-ray light curve, upper limits are not shown and only points with TS $>9$ are plotted.

\subsection{UV-Optical and IR data}

We used the Swift-UV-Optical Telescope (UVOT) for data in the UV and optical bands. They were analyzed using the online tool ${ }^{5}$. To generate the light curve the magnitudes thus obtained and uncorrected for Galactic reddening were then converted to fluxes using the zero points taken from Breeveld et al. (2011). However corrections due to galactic absorption were applied to generate the average data points for SED analysis. In addition to the optical data from Swift-UVOT, we also used optical data in the V-band from both SMARTS and the Steward Observatory, while the IR observations in the $\mathrm{J}$ and K-bands were taken from SMARTS. Optical polarization data whereever available were taken from the Steward Observatory. The details of the instrument and the data reduction procedures for SMARTS can be found in Bonning et al. (2012), while the details on the observations and reductions of data from Steward Observatory can be found in Smith et al. (2009).

\subsection{X-ray Data}

We used data from the X-ray telescope (XRT) on board Swift covering the energy range from $0.3-10 \mathrm{keV}$ (Burrows et al. 2005; Gehrels et al. 2004). The data that spans about 10 years and covering the period August 2008 to August 2018 were taken from the archives at HEASARC ${ }^{6}$. The data were analyzed by the instrument pipleine following standard procedures. For light curve analysis, we used the data collected using both window timing (WT) and photon counting (PC) modes. For spectral analysis of the sources PKS 1510-089, 3C 279 and CTA 102 we used the data collected only from the PC mode, while, for the source 3C 273 we used PC mode data for the quiescent state but for $\gamma$-ray flaring state we used the WT mode data due to the non availability of PC mode data. The XRT data were processed with the xrtpipeline task using the latest CALDB files available with version HEASOFT-6.24. We used the standard grade selection 0-12 and the calibrated and cleaned events were added to generate the energy spectra. For PC mode, we extracted the source spectra from a circular region of radii $60^{\prime \prime}$, and the background spectra were selected from the region of radii $80^{\prime \prime}$ away from the source. In WT mode, for the source we used a circular region of $60^{\prime \prime}$ radii while 

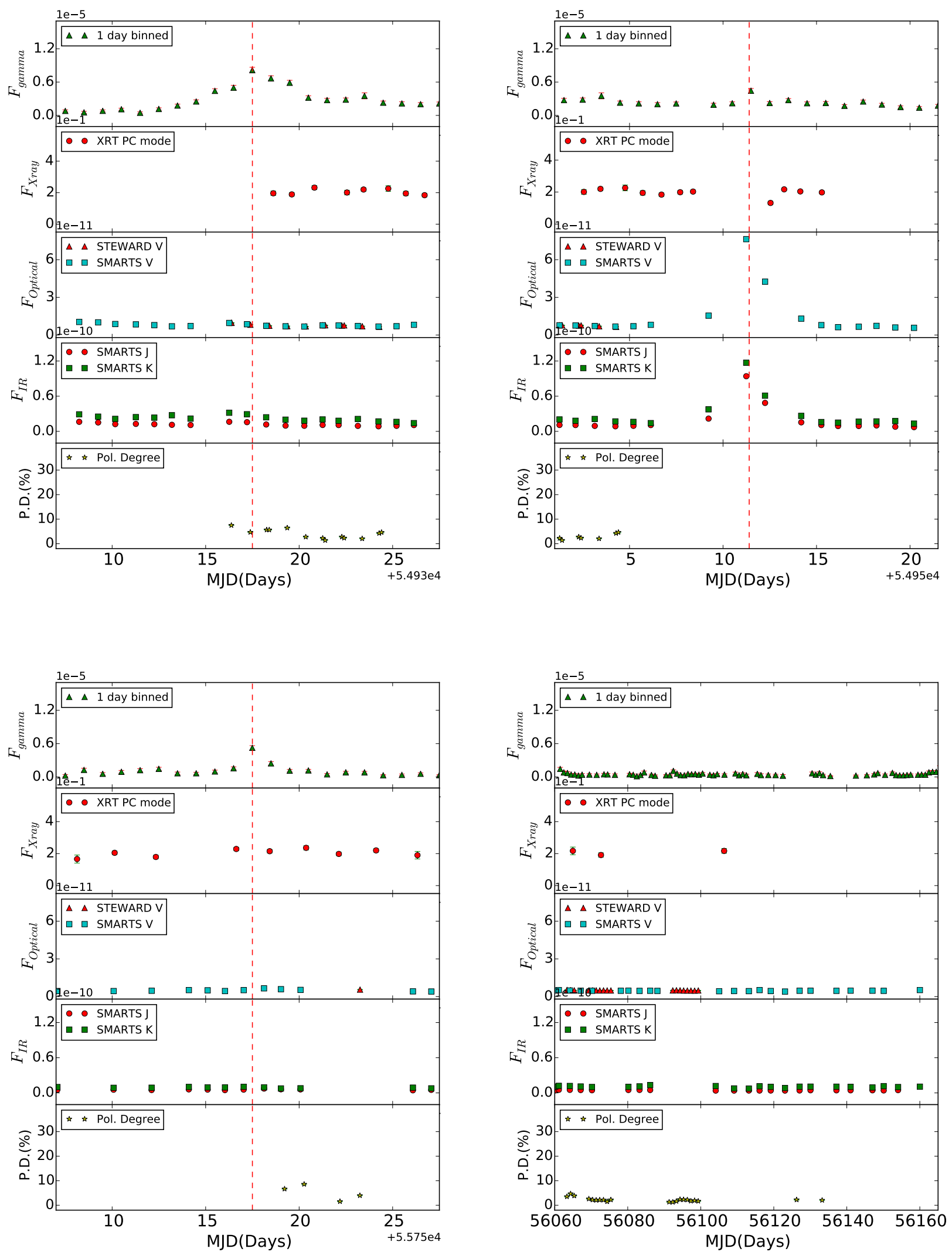

Figure 2. Multi-wavelength light curves for epochs A (top left), B (top right), C (bottom left) and D (bottom right) for the source PKS 1510-089. In all the panels $\gamma$-ray fluxes are in units of $10^{-5} \mathrm{ph} \mathrm{cm}^{-2} \mathrm{~s}^{-1}$. The optical fluxes are in units of $10^{-11} \mathrm{erg} \mathrm{cm}^{-2} \mathrm{~s}^{-1}$ and the IR fluxes are in the units of $10^{-10} \mathrm{erg} \mathrm{cm}^{-2}$ $\mathrm{s}^{-1}$. The vertical dotted line shows the peak of the optical $/ \gamma$-ray flare. 

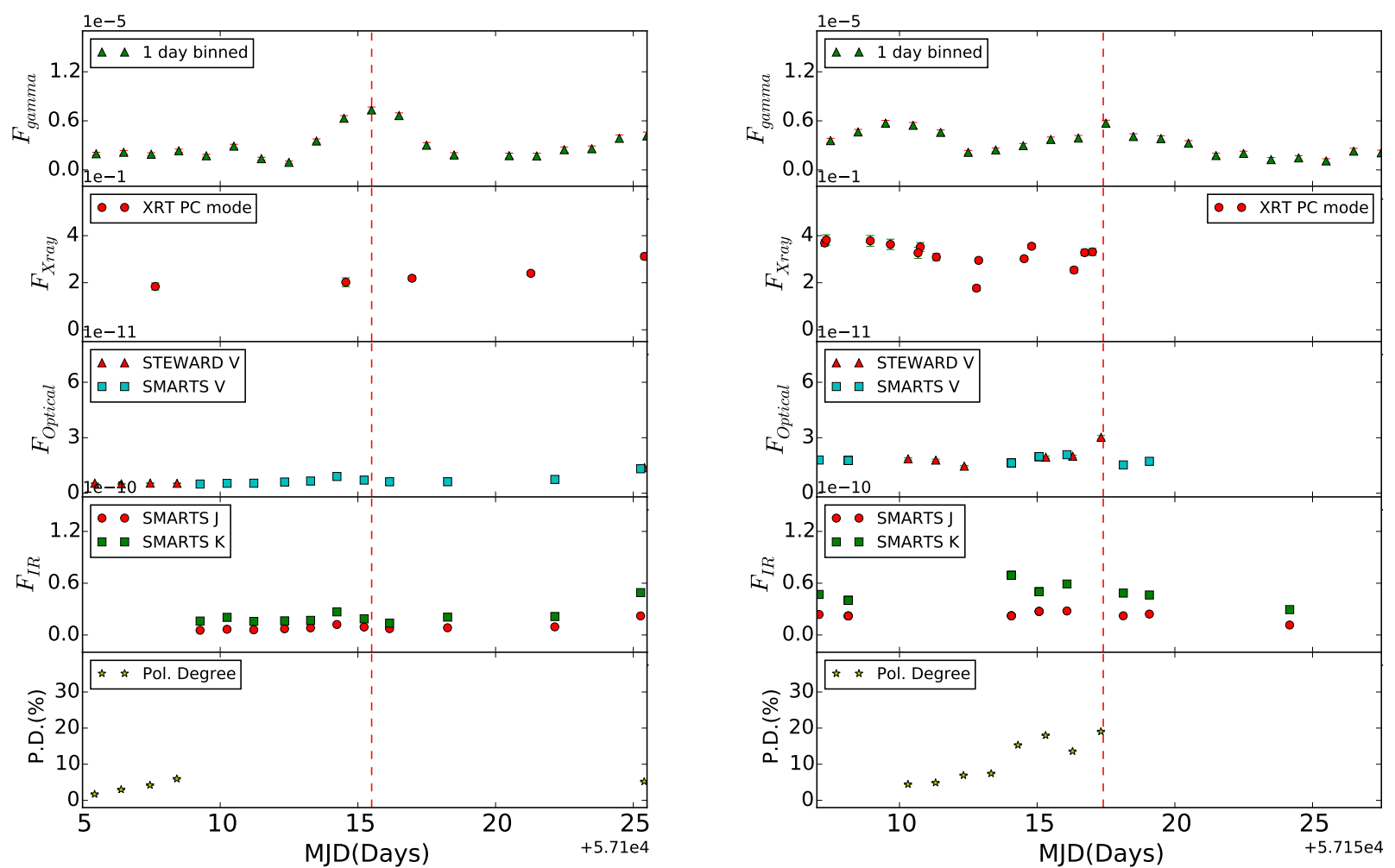

Figure 3. Muti-wavelength light curves for the source PKS 1510-089 during epochs E (left) and F (right). Symbols and lines are as in Fig. 2.

for the background we used the region between $80^{\prime \prime}$ and $120^{\prime \prime}$ radii centered around the source. We combined the exposure maps using XIMAGE and created the ancillary response files using xrtmkarf. For fitting the data within XSPEC (Arnaud 1996), we used an absorbed simple power law model with the Galactic neutral hydrogen column densities of $N_{\mathrm{H}}=6.89 \times 10^{20} \mathrm{~cm}^{-2}, 2.21 \times 10^{20} \mathrm{~cm}^{-2}, 4.81 \times 10^{20}$ $\mathrm{cm}^{-2}$ and $1.68 \times 10^{20} \mathrm{~cm}^{-2}$ from Kalberla et al. (2005) for the sources PKS 1510-089, 3C 279, CTA 102 and 3C 273 respectively. We used $\chi^{2}$ statistics within XSPEC and the uncertainties were calculated at the $90 \%$ confidence level.

\section{$3.3 \gamma$-ray data}

We analyzed 10 years of $\gamma$-ray data from the LAT instrument on board Fermi during the period 08 August 2008 to 08 August 2018 to generate the one day binned $\gamma$-ray light curve. The LAT is an imaging high energy $\gamma$-ray telescope, sensitive in the energy range from $20 \mathrm{MeV}-300 \mathrm{GeV}$. The field of view of the LAT instrument is $20 \%$ of the sky and it scans continuously, covering the whole sky every three hours (Atwood et al. 2009). We used fermipy to analyze the 10 years of $\gamma$-ray data. Fermipy is a python software package that provides a high-level interface for LAT data analysis (Wood et al. 2017). We used Pass 8 data for the analysis where the photon-like events are classified as 'evclass $=128$, evtype $=3$ ' with energy range $0.1 \leqslant \mathrm{E} \leqslant 300 \mathrm{GeV}$. A circular region of interest (ROI) of $15^{\circ}$ was chosen with a zenith angle cut of $90^{\circ}$ in order to remove Earth limb contamination. We used the latest isotropic model "iso_P8R2_SOURCE_V6_v06" and the Galactic diffuse emission model "gll_iem_v06" for the analysis. The recommended criteria "(DATA_QUAL>0)\&\&(LAT_CONFIG==1)" was used for the good time interval selection. In the generation of $\gamma$-ray light curves, we considered the source to be detected if the test statistics (TS) is $>9$ which corresponds to a $3 \sigma$ detection (Mattox et al. 1996).

\section{ANALYSIS}

\subsection{Multi-wavelength light curves}

Analysis for the presence or absence of correlation between optical and $\gamma$-ray flares requires identification of flares in optical and/or $\gamma$ ray light curves. Due to large gaps and/or less number of points in the optical light curves it is not possible to automatically identify epochs (through cross-correlation analysis) on the presence or absence of correlated optical and $\gamma$-ray flux variations. Therefore, flares for detailed analysis were selected visually as follows. Multi-wavelength light curves that span the 10 year period were first generated for each object. In that, optical and $\gamma$-ray light curves were visually inspected for the presence of sharp peaks above their base flux levels. Once identified, expanded multiwavelength light curves were generated for a total duration of 20 days, centered at the optical and/or $\gamma$-ray flares. In an epoch when a $\gamma$-ray flare or an optical flare is identified, we imposed the condition of having data in multiple wavelengths such as $\gamma$-rays, X-rays, UV, optical and IR so as to constrain both the low energy and high energy hump in the SED analysis. These conditions 


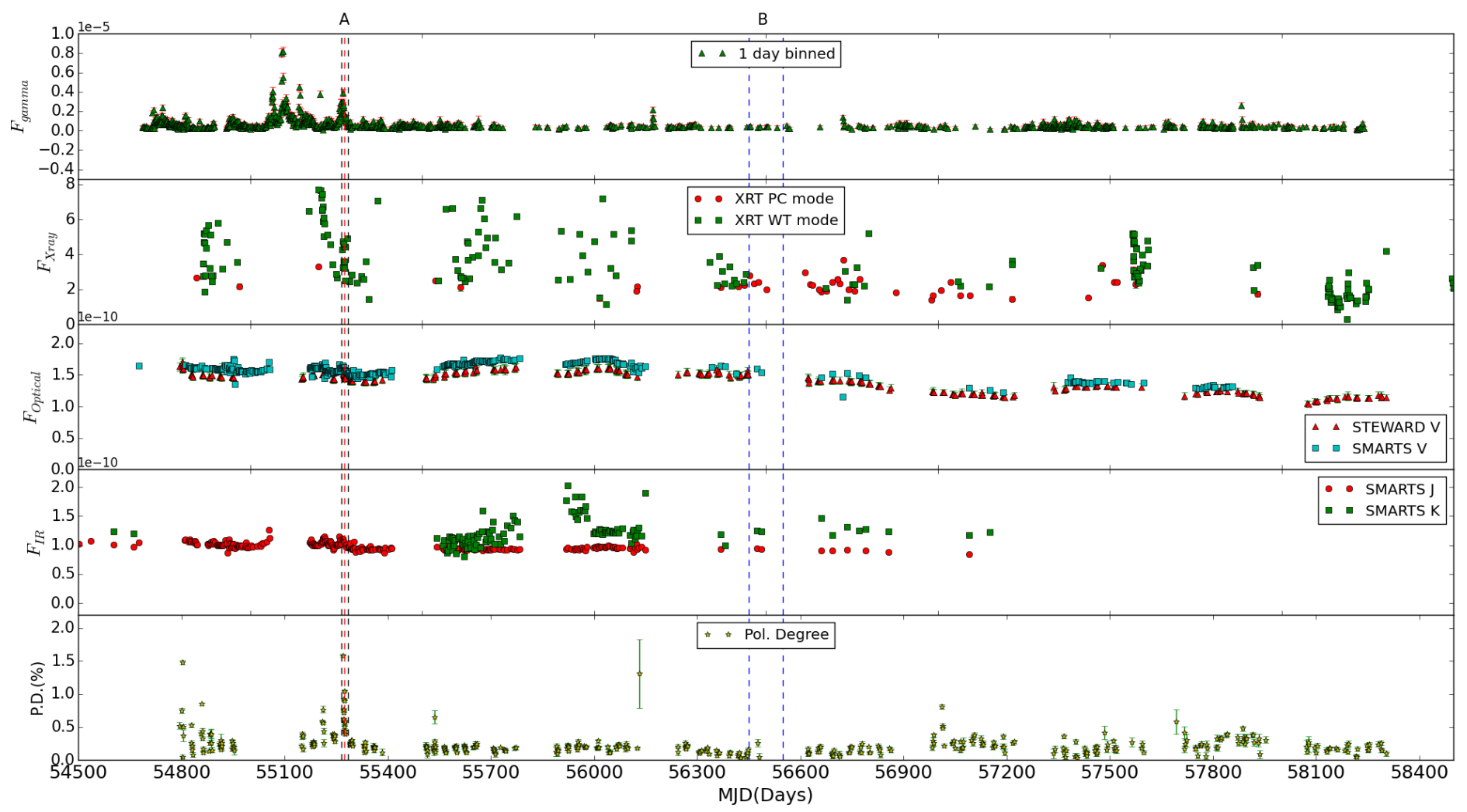

Figure 4. Multi-wavelength light curves of the source 3C 273. The panels and the vertical lines are as in Fig.1

lead to the identification of few flares. Of these we concentrated only on some epochs for each object.

\subsubsection{PKS 1510-089}

The multi-wavelength light curves that include $\gamma$-ray, X-ray, UV, optical and IR are given in Fig. 1. The figure also includes polarization measurements. Inspection of the light curves indicates that the source has displayed varied activity levels that includes both flaring and quiescent periods. From visual inspection of the light curves we selected 6 epochs (A, B, C, D, E and F) in the source for studying the correlations between optical and $\gamma$-ray variations. These epochs were identified by the presence of optical and/or $\gamma$-ray flares in the light curves and a quiescent state in both the optical and $\gamma$-ray bands. A summary of these epochs is given in Table 2 and the multi-wavelength light curves covering a shorter duration during these epochs are shown in Fig. 2 and Fig. 3. More details on these six epochs are given below:

Epoch A: During this epoch, the $\gamma$-ray has increased in flux by a factor of about 10, while the optical and the IR J and K-band fluxes have not shown any variability and are indeed steady. There is also a hint that the X-ray flux from the source is non-variable, however, due to the lack of data during part of the $\gamma$-ray flare, no conclusive statement could be made on the nature of X-ray flux variations. The optical polarization too has not shown noticeable variability during the steady optical/IR brightness state of the source. We conclude that in this epoch we observed a $\gamma$-ray flare with no optical counterpart.

Epoch B: During this epoch, the optical flux has increased by a factor of 6 , while the flux variations in the IR band are at a reduced level. There is also a hint of a very low amplitude $\gamma$-ray flare during the peak of the optical flare, but it is very small. The lack of X-ray data and optical polarization data during the epoch of the optical flare prevents us to make any statement on the nature of X-ray variations as well as the degree of optical polarization during this epoch. Thus in this epoch the source has shown correlated optical and $\gamma$-ray flux variations, though the amplitude of variations in the $\gamma$-ray band is much lower than that of the optical and IR bands.

Epoch C: The flux variations noticed in this epoch is similar to that observed during epoch A. A minor flare is observed in the $\gamma$-ray band, but the source is stable in the X-ray, optical and IR bands. We do not have optical polarization data during the $\gamma$-ray flare for an idea on the nature of optical V-band polarization. Thus, during this epoch, the source has shown a $\gamma$-ray flare without a counterpart in the low energy X-ray, optical and IR bands.

Epoch D: During this period, the source is in the quiescent state in all the energy bands analyzed here.

Epoch E: During this epoch, the source has shown a strong $\gamma$-ray flare, however, such a flare is not seen in the X-ray, optical and IR bands. Here too, optical polarization data is not available during the period of the $\gamma$-ray flare. Thus, in this epoch, the source has shown 

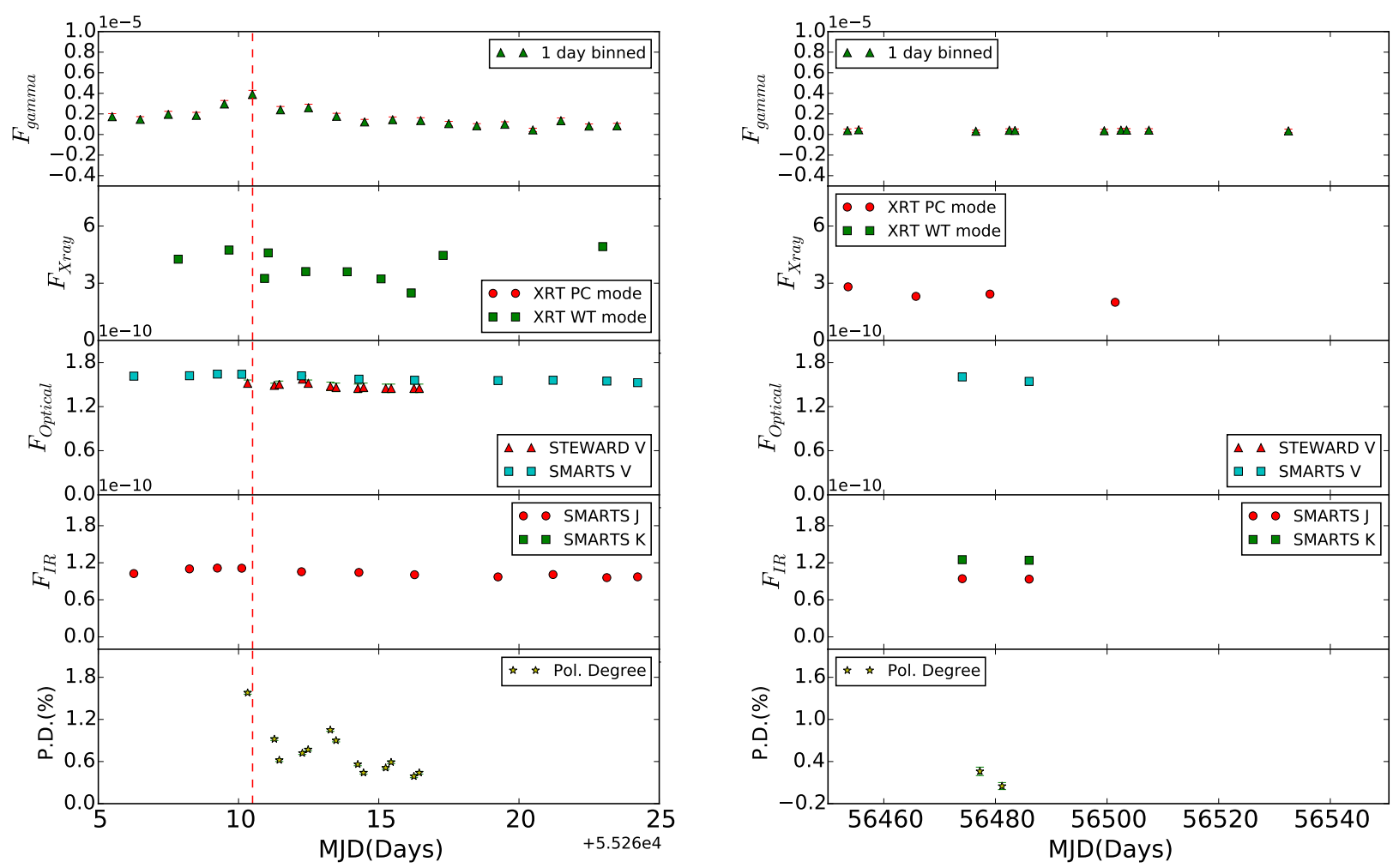

Figure 5. Light curves of 3C 273 for epoch A (left panel) and epoch B (right panel). The $\gamma$-ray fluxes are in units of $10^{-5} \mathrm{ph} \mathrm{cm}^{-2} \mathrm{~s}^{-1}$ and the optical and IR fluxes are in units of $10^{-10} \mathrm{erg} \mathrm{cm}^{-2} \mathrm{~s}^{-1}$. The vertical dotted line shows the peak of the $\gamma$-ray flare.

a $\gamma$-ray flare without similar flaring in the other wavelengths such as X-rays, optical and IR.

Epoch F: A weak $\gamma$-ray flare is seen in the source during this period. Simultaneous to the $\gamma$-ray flare, there is an indication of a minor optical flare which is also accompanied by an increase in the degree of optical polarization. There is paucity of X-ray and IR data during the peak of the $\gamma$-ray flare. Thus, the optical and $\gamma$-ray flux variations are closely correlated during this epoch.

\subsection{2 $3 C 273$}

We show in Fig. 3 the multi-wavelength light curves for the source $3 \mathrm{C}$ 273. The source is mostly quiescent during the period 2008 to 2018 August except for few instances where it has flared in the $\gamma$-ray band. We identified two epochs in this source for studying the correlation between optical and $\gamma$-rays. The details of these two epochs are given in Table 2. They are also marked in Fig. 4, and an expanded version of these two epochs is given in Fig. 5.

Epoch A: There is a prominent $\gamma$-ray flare during this epoch, wherein the $\gamma$-ray flux has increased by a factor of two at the peak of the $\gamma$-ray flare. During the peak of the $\gamma$-ray flare, the X-ray, optical and IR brightness do not show significant changes. Also, the source lacks optical polarization data during the peak of the $\gamma$-ray flare. The source has thus shown a $\gamma$-ray flare without an optical counterpart in this epoch.

Epoch B: During this epoch the source is found to be in the quiescent state in all the wavebands namely $\gamma$-rays, X-ray, optical and IR. The source is also weakly polarized in the optical V-band during this period.

\subsection{3 $3 C 279$}

We show in Fig. 6 the multi-wavelength light curves. From visual inspection, we identified four epochs in this source for studying the correlation between optical and $\gamma$-ray flux variations. We give in Table 2 the summary of those four epochs. An expanded view of the multi-wavelength flux variations in the source is shown in Fig. 7. Below, we summarize the salient aspects of these four epochs.

Epoch A: In this epoch the source is in the quiescent state. In the one day binned $\gamma$-ray light curve the source is below the detection limit for many days during this 100 days period. Also, in the X-ray, optical and IR bands, the source is non-variable during this period. However, during the middle of this epoch, the optical polarization increased by a factor of about 5 from $\sim 6 \%$ to $\sim 25.8 \%$. During this period of increased optical polarization, the source did not show flux variations in any of the bands.

Epoch B: During this epoch, the source has shown a minor flare in the $\gamma$-ray band with no corresponding flare in the optical, IR and $\mathrm{X}$-ray bands. Polarization data is not available during the peak of the $\gamma$-ray flare thereby making it impossible to know the polarized nature of the source. By comparing the multi-wavelength light curves during this epoch, we conclude that the source showed a $\gamma$-ray flare without an optical counterpart.

Epoch C: During this epoch, a strong $\gamma$-ray flare was observed wherein the $\gamma$-ray flux increased by a factor of about 3. During the 


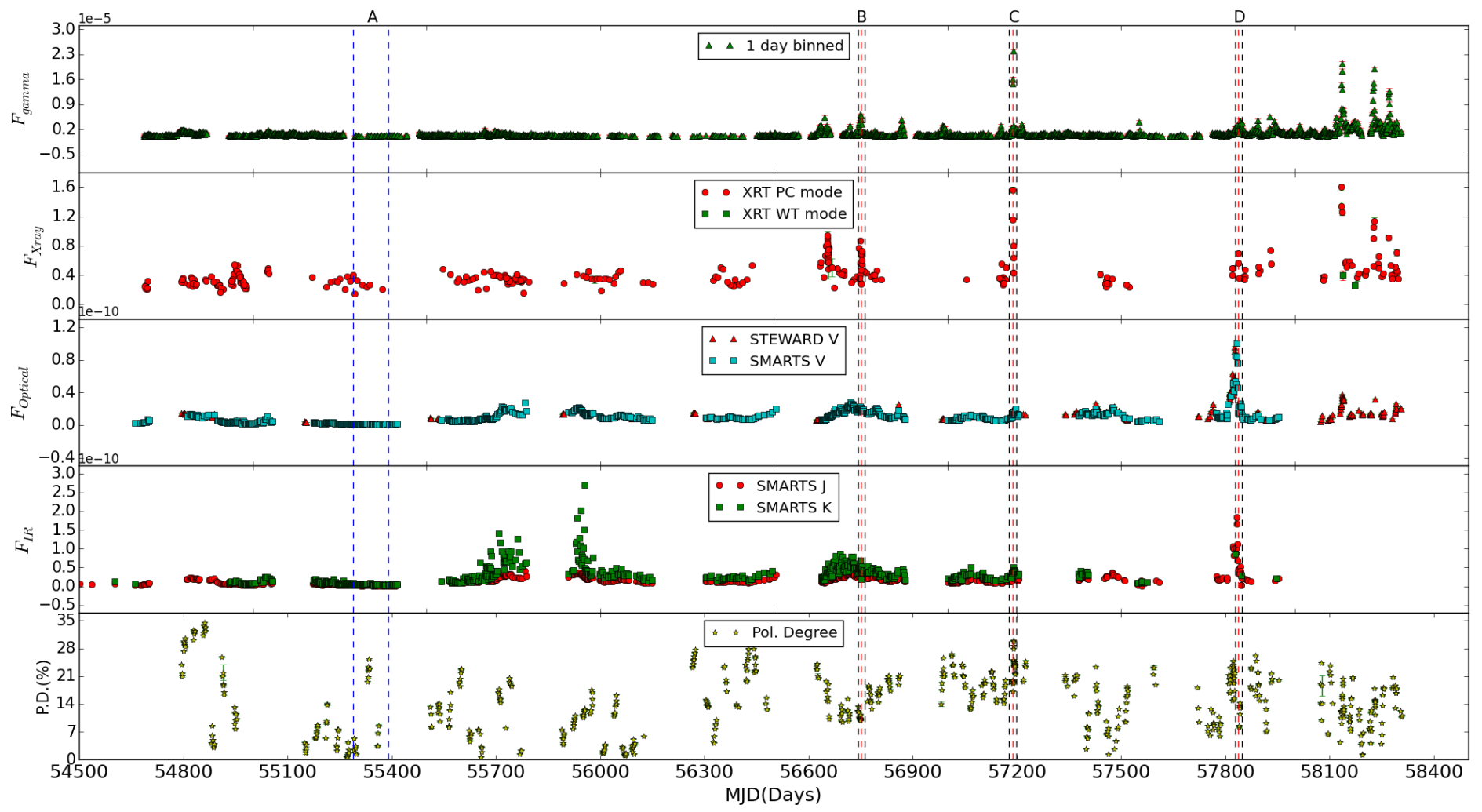

Figure 6. The light curves of the source 3C 279 in different wavelengths. The panels and the dashed lines are as in Fig. 1.

peak of the $\gamma$-ray flare, X-ray too showed a flare, however, in the optical and IR bands, the source was found to be stable with no signs of flux variability. An interesting behaviour displayed by this source is an apparent negative correlation of $\gamma$-ray and X-ray flux variations to the optical polarization. During the epoch when the $\gamma$-ray and $\mathrm{X}$-ray were at their peaks, the optical polarization was low, and it gradually increased when the X-ray and $\gamma$-ray fluxes declined.

Epoch D: During this epoch, the source showed a prominent optical and IR flare. The flare was found be be asymmetric with a fast rise and slow decay. During the epoch of the optical and IR flare the source did not show any variation in the $\gamma$-ray band. Due to the lack of polarization data during the peak of the optical and IR flare, we could not make any statement on the optical polarization state during the time of the optical and IR flare. Thus this epoch is a clear example of the source showing an optical flare without a $\gamma$-ray counterpart.

\subsubsection{CTA 102}

The source was found to be in a steady and low brightness state during most of the time between 2008 August to 2018 August, except for a spectacular $\gamma$-ray flare in the beginning of 2016. The multiwavelength light curves are shown in Fig. 8. We have identified 2 epochs in this source for studying the correlation between optical and $\gamma$-ray variations. A summary of these two epochs is given in Table 2 and expanded plots of these two epochs are shown in Fig. 9. More details on these two epochs are given below.
Epoch A: During this epoch the source was in the quiescent state in all the wavebands considered in this work.

Epoch B: The source showed a major $\gamma$-ray flare during this epoch. This flaring in the $\gamma$-ray band was also accompanied by flaring behaviour in the X-ray and optical wavelengths. The nature of IR flux during this period is uncertain due to the non-availability of IR data during this flaring period. Thus, during this epoch, the source showed correlated flux variations in the optical and $\gamma$-ray bands.

\subsection{Spectral Variations}

Blazars show spectral variations in addition to flux variations. To characterize the spectral variability of the blazars studied here, we looked for variations in the V-J color against the V-band brightness. This spectral analysis was done for all the epochs in the sources PKS 1510-089, 3C 273 and 3C 279 for which correlations between optical and $\gamma$-ray flux variations were explored in Section 4.1. Such spectral analysis was not carried out for the source CTA 102 due to the lack of J-band data. Spectral variations were characterized by linear least squares fit to the colour-magnitude diagram by taking into account the errors in both the colour and magnitude. A source is considered to have shown colour variation if the Spearman rank correlation coefficient is $>0.5$ as well as $<-0.5$ with the null hypothesis probability $\mathrm{P}<0.05$. In the source PKS $1510-089$, we found a "redder when brighter" (RWB) trend at epochs A and E, however, during epoch $\mathrm{F}$ we observed a "bluer when brighter" (BWB) 

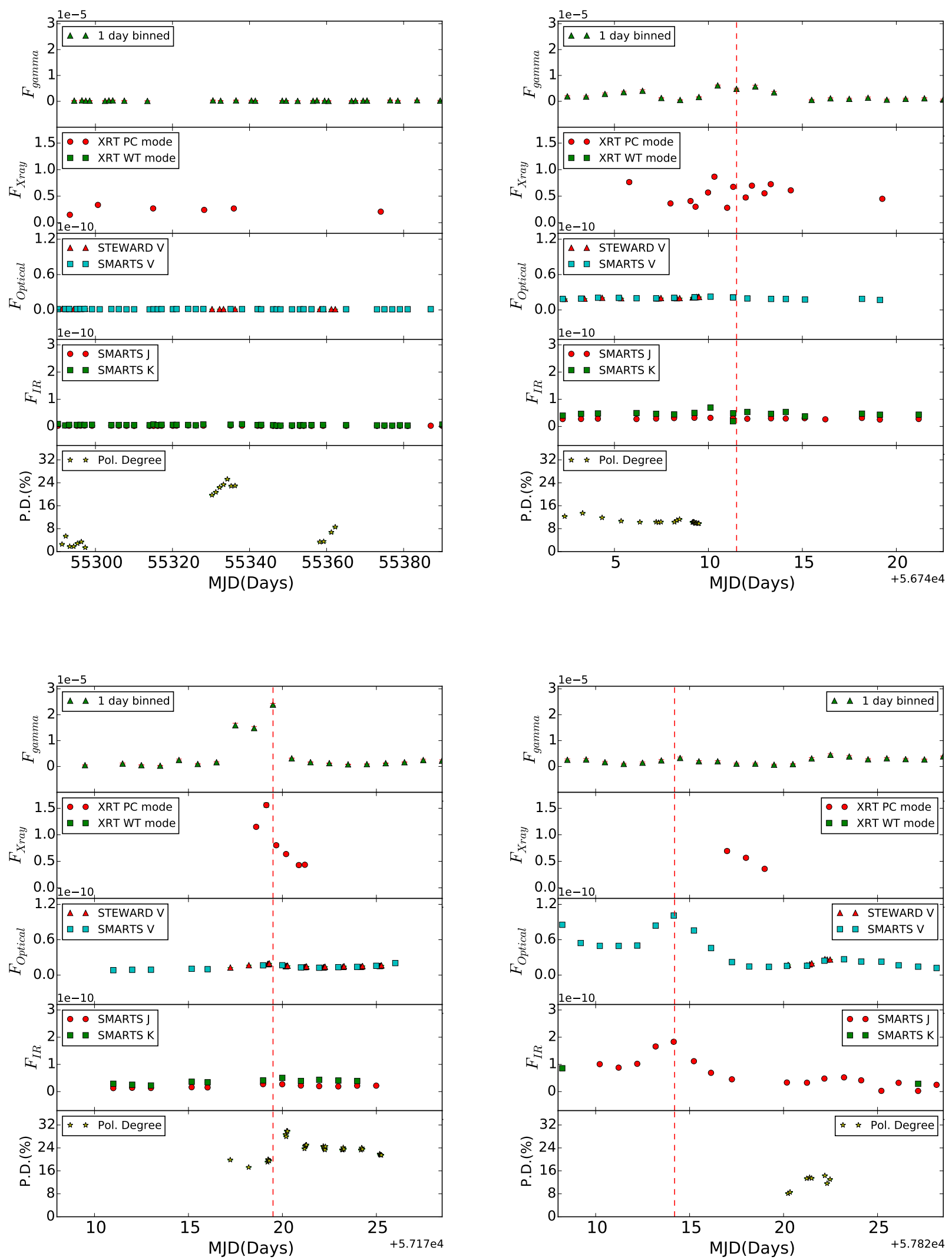

Figure 7. Multi-wavelength light curves of the source 3C 279 for epoch A (top left), epoch B (top right), epoch C (bottom left) and epoch D (bottom right). The optical and IR light curves have units of $10^{-10} \mathrm{erg} \mathrm{cm}^{-2} \mathrm{~s}^{-1}$, while the $\gamma$-ray light curves have units of $10^{-5} \mathrm{ph} \mathrm{cm}^{-2} \mathrm{~s}^{-1}$. The dashed lines indicate the peak of the optical $/ \gamma$-ray flare. 


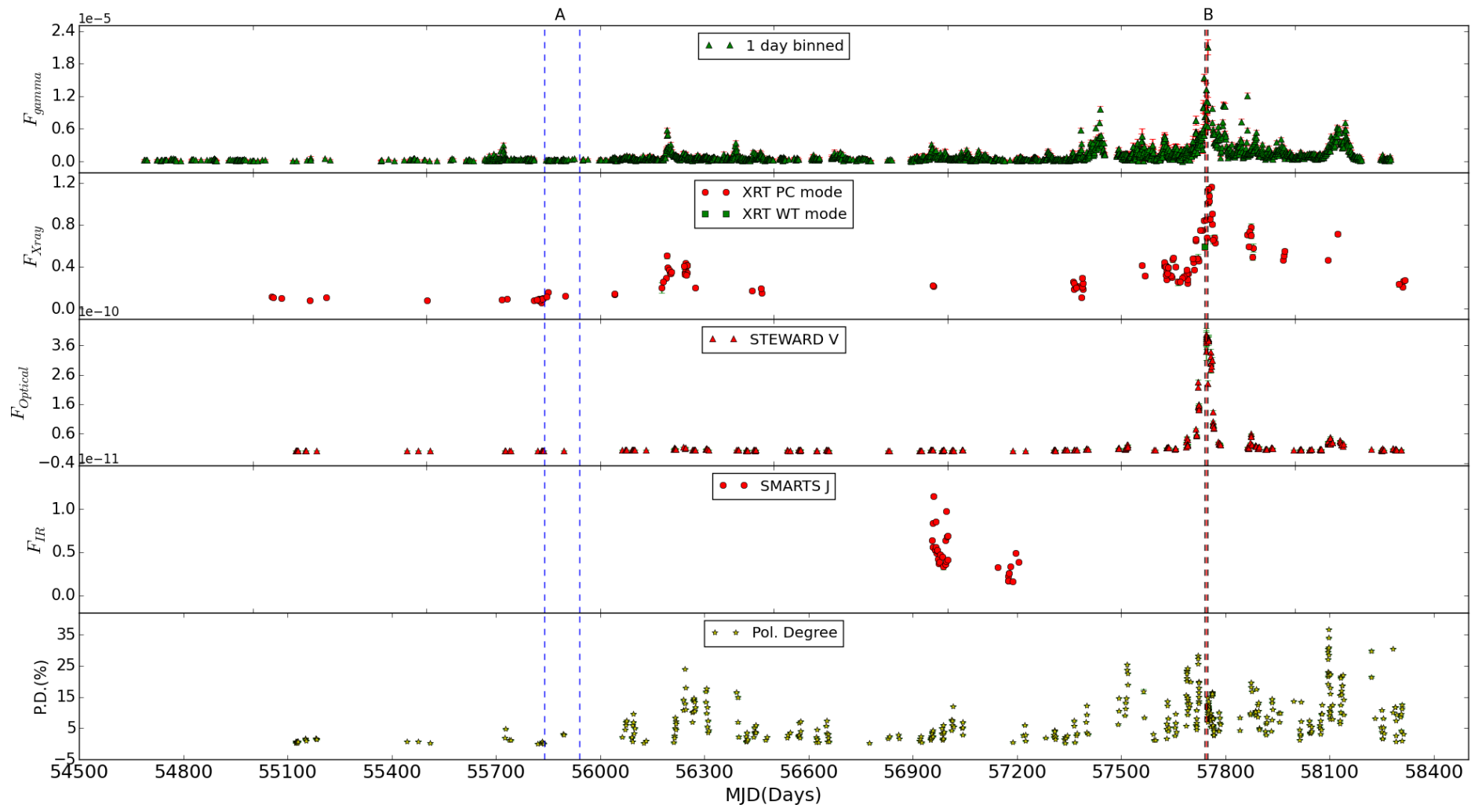

Figure 8. Long term light curves of the source CTA 102 in different wavelengths. Details in this figure are similar to that of Fig. 1.

behaviour. In the source $3 \mathrm{C} 273$, during epoch $\mathrm{A}$, we found the source to show a RWB trend. For the source $3 \mathrm{C} 279$, we found BWB behaviour during epochs $\mathrm{B}$ and $\mathrm{D}$. This indicates that the spectral variations shown by FSRQs are complex and a FSRQ may not show the same spectral variability pattern at all times. The colour magnitude diagram for the sources PKS 1510-089, 3C 273 and 3C 279 are shown in Fig. 10.

\section{$4.3 \gamma$-ray spectra}

To study the intrinsic distribution of electrons in the jets that are involved in the $\gamma$-ray emission process, we generated $\gamma$-ray spectra for all the selected epochs (as detailed in Section 4.1) of the four sources. We fitted the $\gamma$-ray spectra with the two models namely (i) the simple power law (PL) model and (ii) the log-parabola (LP) model. For PL model we used the following

$d N(E) / d E=N_{\circ}\left(E / E_{\circ}\right)^{\Gamma_{P L}}$

where $N_{\circ}$ is the normalization of the energy spectrum and $E_{\circ}$ is the scaling factor and $\Gamma_{P L}$ is the photon index.

The LP model has the following form (Nolan et al. 2012)

$d N(E) / d E=N_{\circ}\left(E / E_{\circ}\right)^{-\alpha-\beta \ln \left(E / E_{\circ}\right)}$

where, $\mathrm{dN} / \mathrm{dE}$ is the number of photons in $\mathrm{cm}^{-2} \mathrm{~s}^{-1} \mathrm{MeV}^{-1}, \alpha$ is photon index at $E_{\circ}, \beta$ is the curvature index that defines the curvature around the peak, $\mathrm{E}$ is the energy of the $\gamma$-ray photon, $\mathrm{N}_{\circ}$ is the normalization and $\mathrm{E}_{\circ}$ is the scaling factor.
To test the model that well describes the $\gamma$-ray spectra (PL against LP), as well as the presence of curvature, we used the maximum likelihood estimator gtlike. Following Nolan et al. (2012), we calculated the curvature of the test statistics as $T S_{\text {curve }}=2\left(\log L_{L P}\right.$ - $\left.\log L_{P L}\right)$. Here L represents the likelihood function. We used the threshold $T S_{\text {curve }}>16$ for the presence of a statistically significant curvature in the $\gamma$-ray spectra, (at the $4 \sigma$ level; Mattox et al. 1996). We found that for all the epochs, the $\gamma$-ray spectra is well fit by the LP model except for the quiescent epoch of the source 3C 273 which is well fit by the PL model. The results of the $\gamma$-ray spectral analysis are given in Table 3. One example of the PL model that best fits the data (for the source 3C 273 at Epoch B) and another example of a LP model that best fits the data (for the source 3C 279 at Epoch C) is shown in Fig. 11.

\subsection{Spectral energy distribution modelling}

The sources studied here showed various characteristics in their optical and $\gamma$-ray flux variations. There are instances when (a) optical and $\gamma$-ray flux variations are correlated, (b) there is an optical flare without a $\gamma$-ray counterpart and (c) there is a $\gamma$-ray flare without an optical counterpart. To further characterize the nature of the sources during the various epochs, we constructed their broad band SED during these epochs and studied them using simple one zone leptonic emission model. To obtain the SEDs in UV, optical and IR, all photometric measurements during each epoch were averaged filter wise to 

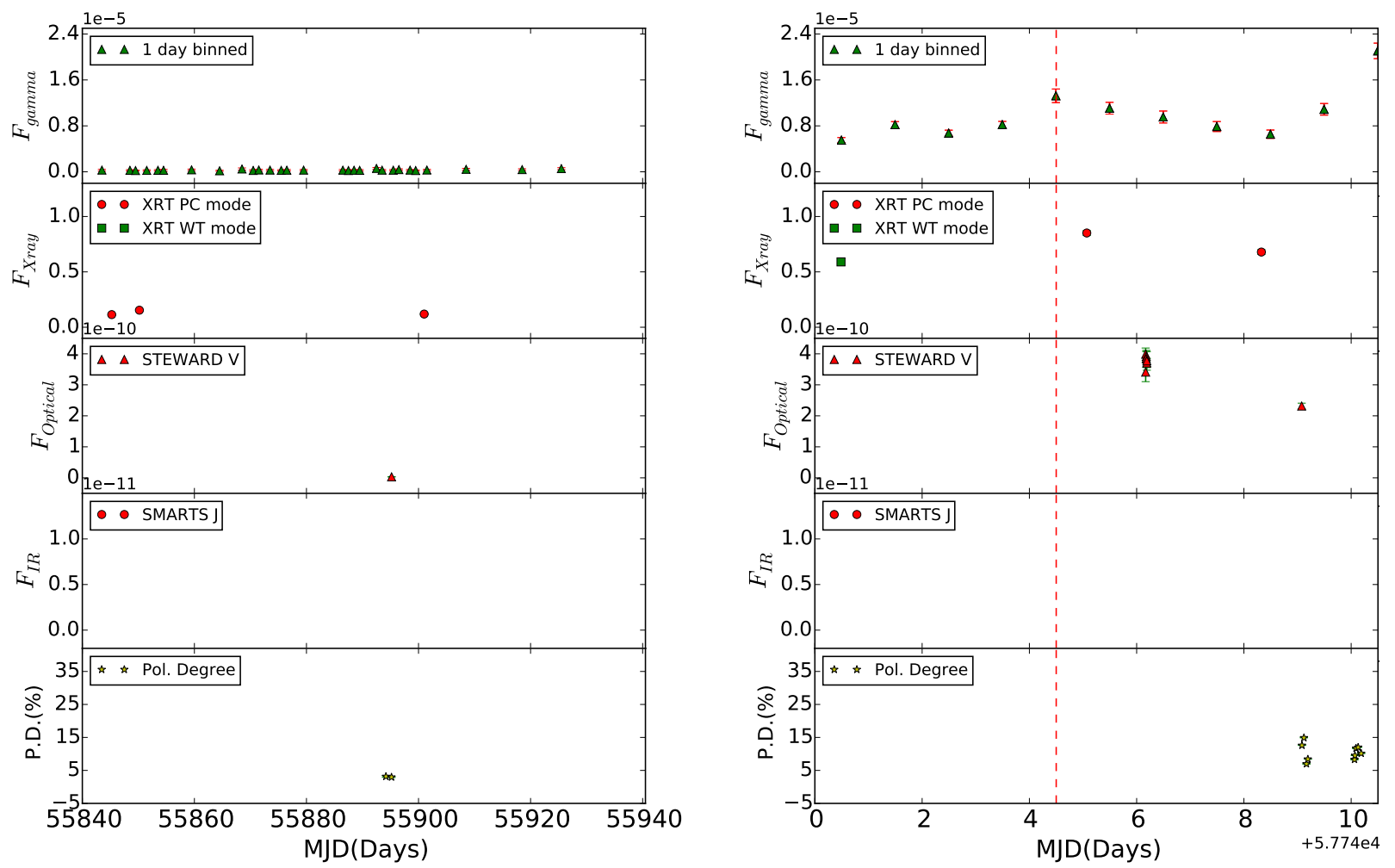

Figure 9. The left and right panels show the multi-wavelength light curves of the source CTA 102 for epoch A and B respectively. The dashed line shows the peak of the $\gamma$-ray flare. The optical and $\gamma$-ray fluxes are in units of $10^{-10} \mathrm{erg} \mathrm{cm}^{-2} \mathrm{~s}^{-1}$ and $10^{-5} \mathrm{ph} \mathrm{cm}^{-2} \mathrm{~s}^{-1}$ respectively.

Table 3. Details of the PL (Eq. 1) and LP (Eq. 2) model fits for the different epochs of the sources PKS 1510-089, 3C 273, 3C 279 and CTA 102. $\Gamma_{P L}$ is the photon index from PL fitting, $\alpha$ and $\beta$ the photon index and curvature index from LP fit to the spectra, TS is the test statistics, Log L is the log-likelihood, and $T S_{\text {curve }}$ is the curvature of the test statistics defined as 2(log $\left.L_{L P}-\log L_{P L}\right)$. For all the epochs in the sources PKS 1510-089, 3C 279 and CTA 102 and the epoch A of 3C 273, LP model best describes the spectra, while the spectra during epoch B of 3C 273 is well fit by the PL model.

\begin{tabular}{|c|c|c|c|c|c|c|c|c|c|c|}
\hline \multirow[b]{2}{*}{ Epochs } & \multicolumn{4}{|l|}{ PL } & \multicolumn{5}{|c|}{ LP } & \multirow[b]{2}{*}{$\mathrm{TS}_{\text {curve }}$} \\
\hline & $\Gamma_{P L}$ & Flux & TS & $-\log L$ & $\alpha$ & $\beta$ & Flux & TS & $-\log L$ & \\
\hline \multicolumn{11}{|c|}{ PKS 1510-089 } \\
\hline A & $-2.41 \pm 0.04$ & $0.08 \pm 0.006$ & 1502.89 & 32666.94 & $2.35 \pm 0.03$ & $0.10 \pm 0.02$ & $2.89 \pm 0.08$ & 6626.5 & 30287.04 & 4759.8 \\
\hline B & $-2.38 \pm 0.01$ & $1.10 \pm 0.02$ & 4484.27 & 40710.69 & $2.31 \pm 0.04$ & $0.05 \pm 0.02$ & $2.30 \pm 0.03$ & 6046.02 & 39865.06 & 1691.24 \\
\hline $\mathrm{C}$ & $-2.29 \pm 0.05$ & $1.05 \pm 0.03$ & 2143.16 & 26980.49 & $2.24 \pm 0.06$ & $0.06 \pm 0.04$ & $1.16 \pm 0.01$ & 2149.74 & 26918.59 & 123.8 \\
\hline $\mathrm{D}$ & $-2.46 \pm 0.01$ & $0.36 \pm 0.007$ & 1396.18 & 71986.58 & $2.40 \pm 0.05$ & $0.009 \pm 0.007$ & $0.38 \pm 0.009$ & 1383.28 & 71943.76 & 85.64 \\
\hline E & $-2.35 \pm 0.02$ & $0.10 \pm 0.006$ & 3632.06 & 41998.12 & $2.21 \pm 0.04$ & $0.05 \pm 0.02$ & $3.00 \pm 0.02$ & 12254.5 & 37916.38 & 8163.48 \\
\hline $\mathrm{F}$ & $-2.26 \pm 0.02$ & $0.44 \pm 0.008$ & 7069.12 & 33739.65 & $2.18 \pm 0.02$ & $0.06 \pm 0.01$ & $3.05 \pm 0.05$ & 11891.14 & 31131.89 & 5215.52 \\
\hline \multicolumn{11}{|l|}{3 C 273} \\
\hline A & $-2.48 \pm 0.04$ & $0.03 \pm 0.003$ & 384.26 & 18366.24 & $2.45 \pm 0.06$ & $0.02 \pm 0.04$ & $0.49 \pm 0.01$ & 1965.47 & 17796.95 & 1138.58 \\
\hline B & $-2.91 \pm 0.10$ & $0.005 \pm 1.13$ & 22.10 & 53603.31 & $2.91 \pm 0.11$ & $0.13 \pm 0.16$ & $0.46 \pm 0.02$ & -328.63 & 53791.17 & -375.72 \\
\hline \multicolumn{11}{|l|}{$3 \mathrm{C} 279$} \\
\hline A & $-2.42 \pm 0.03$ & $0.02 \pm 0.003$ & 193.899 & 61574.51 & $2.46 \pm 0.07$ & $0.06 \pm 0.01$ & $0.16 \pm 0.004$ & 341.966 & 61472.92 & 203.18 \\
\hline B & $-2.28 \pm 0.04$ & $0.29 \pm 0.003$ & 5059.59 & 28075.17 & $2.13 \pm 0.05$ & $0.09 \pm 0.02$ & $0.61 \pm 0.03$ & 6630.85 & 27337.68 & 1474.98 \\
\hline $\mathrm{C}$ & $-2.22 \pm 0.00$ & $0.60 \pm 0.01$ & 14113.31 & 41165.00 & $2.05 \pm 0.03$ & $0.10 \pm 0.01$ & $4.09 \pm 0.001$ & 25625.3 & 37399.62 & 7530.76 \\
\hline $\mathrm{D}$ & $-2.28 \pm 0.03$ & $0.16 \pm 0.003$ & 2426.62 & 24463.13 & $2.14 \pm 0.04$ & $0.08 \pm 0.02$ & $1.44 \pm 0.01$ & 5391.43 & 23282.19 & 2361.88 \\
\hline \multicolumn{11}{|l|}{ CTA 102} \\
\hline A & $-2.49 \pm 0.00$ & $0.002 \pm 0.91$ & 26.02 & 57614.41 & $2.58 \pm 0.06$ & $0.02 \pm 0.05$ & $0.16 \pm 0.004$ & 565.75 & 57358.15 & 512.52 \\
\hline B & $-2.12 \pm 0.004$ & $0.36 \pm 0.008$ & 10266.7 & 35236.65 & $1.84 \pm 0.03$ & $0.09 \pm 0.01$ & $0.63 \pm 0.02$ & 14952.58 & 35028.19 & 416.92 \\
\hline
\end{tabular}



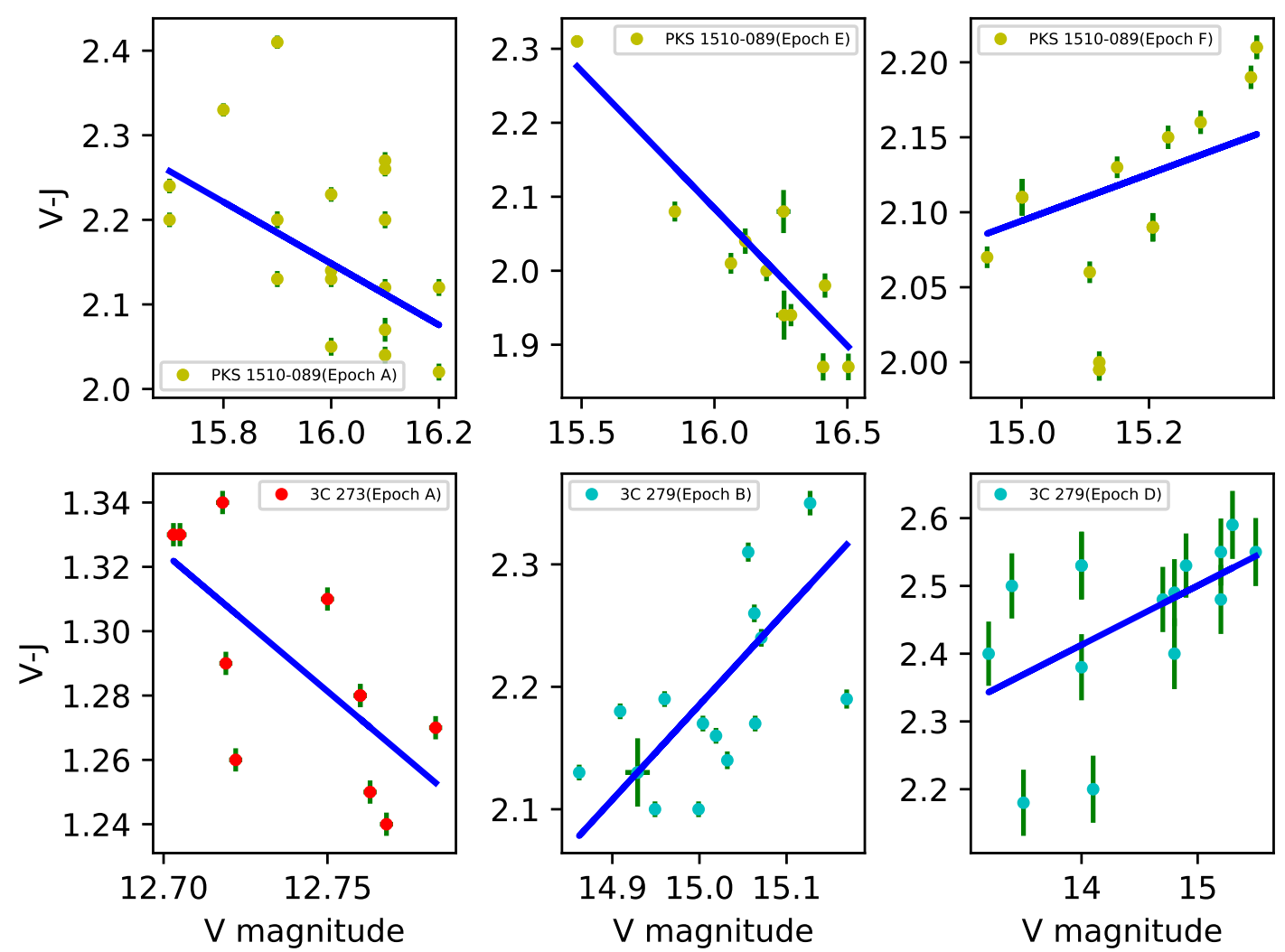

Figure 10. Colour-magnitude relations. From the left, the top panels are for the source PKS 1510-089, epochs A, E and F. In the bottom the left panel is for the epoch A of 3C 273, while the other two panels are for the epochs B and D of 3C 279.
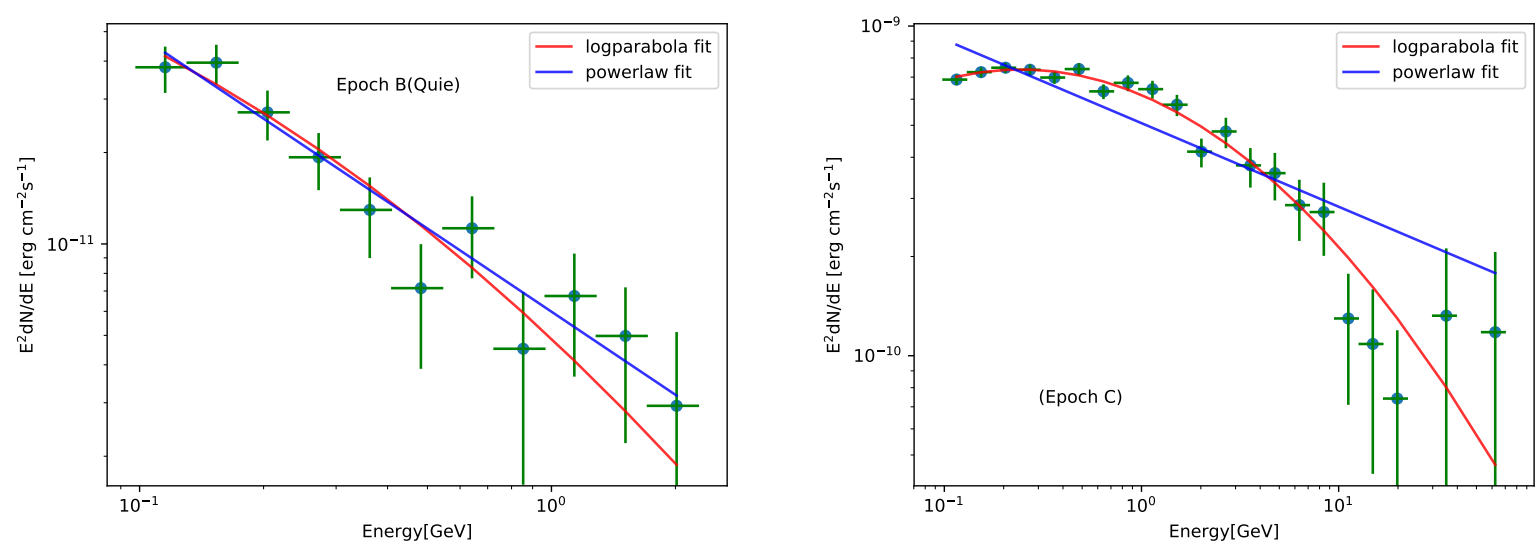

Figure 11. Observed and model fits to the $\gamma$-ray spectra of the source 3C 273 for epoch B (left panel) and of 3C 279 for epoch C (right panel).

get one photometric point per epoch. In the case of X-ray and $\gamma$-ray bands, all the data in each epoch were used to generate the average $\mathrm{X}$-ray and $\gamma$-ray spectra.

Modelling the time averaged spectral energy distribution during various epochs under different emission mechanisms can help us to understand the physical conditions in the source. The $\gamma$-ray emission from FSRQs is generally interpreted as the external Compton scat- tering of thermal IR photons (Shah et al. 2019; Rajput et al. 2019) and hence the broad band SEDs are modeled using synchrotron, synchrotron self Compton and external Compton emission processes. The details of the model are given in Sahayanathan et al. (2018) and the best fit physical parameters of the sources are obtained by fitting the broad band SED using $\chi^{2}$ minimization technique. We added $12 \%$ systematics to the data in order to account for the emission 
model related uncertainties. There are twelve free parameters in our model, of which six parameters govern the electron energy distribution, namely electron energy index before the break (p), electron energy index after the break $(\mathrm{q})$, the break Lorentz factor $\left(\gamma_{b}\right)$, minimum Lorentz factor of the electron distribution $\left(\gamma_{\min }\right)$, the maximum Lorentz factor of the electron distribution $\left(\gamma_{\max }\right)$ and the electron energy density $\left(U_{e}\right)$. The other six parameters in the model are the magnetic field (B), size of the emission region (R), Lorentz factor $(\Gamma)$, jet viewing angle $(\theta)$, external photon field temperature $(\mathrm{T})$ and the fraction of the external photons that take part in the EC process (f). In order to investigate the different flaring behaviour between optical and $\gamma$-ray, firstly we fitted the quiescent epoch to obtain the parameters for all the sources. From the observed SED, we could obtain the high and low energy spectral indices, the synchrotron flux in the optical, the SSC and EC fluxes in the X-ray and $\gamma$-ray energies respectively. Consistently for the model fit, we chose five free parameters namely p, q, $U_{e}, \Gamma$ and B while the other parameters were frozen to typical values. The adopted values of the seven frozen parameters for the four sources are given in Table 4. In Table 5 and Figure 12 to Figure 16, we summarize the results of the fitting. From the residuals given in the bottom panel of the Figures for some sources, it is clear the optical data is steeper and deviate significantly from the best fit model. This suggests the presence of more than one emission component at optical/UV energies.

To account for the deviation of the model from the optical spectra, we modified the model by including the emission from the accretion disk. The thermal emission from the disk is decided by two parameters, namely the central black hole mass and the mass accretion rate (Shakura \& Sunyaev 1973; Jolley \& Kuncic 2008). The mass of the black hole is obtained from Chen (2018) and the accretion rate is fitted to reproduce the optical spectra. This procedure significantly improved the resultant $\chi^{2}$ and the best fit parameters are given in Table 5. The model spectrum along with the observed data are given in Fig. 12 to 16 . Through this exercise we also demonstrate the capability to extract the accretion disk component from the broadband SED through a realistic spectral modelling involving different emission mechanisms.

\section{RESULTS AND DISCUSSION}

\section{$5.1 \gamma$-ray spectra}

The high energy $\gamma$-ray spectra of FSRQs and low synchrotron peaked BL Lacs deviate from the power law behaviour and are phenomenologically better represented either as a broken power law (BPL) or a LP model. Such departures from simple PL fits noted as a common feature in FSRQs firstly in the early observations from Fermi-LAT (Abdo et al. 2010b) are now observed in the high energy spectra of several FSRQs (Harris et al. 2014; Paliya et al. 2015; Rajput et al. 2019; Sahakyan 2020). The cause of the spectral curvature seen in the $\gamma$-ray spectra from Fermi-LAT is still not known. Several scenarios, both intrinsic and extrinsic origins are proposed in the literature to explain the break in the $\gamma$-ray spectrum of FSRQs.

One of the causes could be due to the attenuation of $\gamma$-rays by photon-photon pair production within the BLR due to HeII recombination and $\mathrm{HI}$ recombination. In this scenario termed as the double absorber model (Poutanen \& Stern 2010), one expects to see a break around 4-7 GeV and another break around 19.2-30 GeV. Such an observation would imply absorption of $\gamma$-rays by BLR photons and the $\gamma$-ray production site must lie within the BLR. However, observations do not support the double absorber model (Harris et al. 2012).
Alternatively, the break in the $\mathrm{GeV}$ spectra of FSRQs can happen by Klein-Nishina effect on the inverse Compton scattering of BLR photons by relativistic jet electrons with a curved distribution (Cerruti et al. 2013). But, from an analysis of the $\gamma$-ray spectra of a large number of blazars, Costamante et al. (2018) found that in FSRQs, the observed $\gamma$-ray spectra is not by IC scattering of BLR photons and the $\gamma$-ray emission site lies outside the BLR.

Apart from the above, the break in the $\gamma$-ray spectra of FSRQs can also happen due to intrinsic effects because of the electrons in the relativistic jets of these sources either having a cut-off in their energy distribution or a log-parabola energy distribution. In this work, the SEDs of all the sources in the different epochs are well modelled by IC scattering of the photons from the obscuring torus, and the $\gamma$-ray emission region lies outside the BLR where IC takes place in the Thomson regime. The results of the $\gamma$-ray spectral analysis carried out on all the epochs in the objects and reported in Table 3 . We note that in the 3FGL catalog (https://www.ssdc.asi.it/fermi3fgl/), the $\gamma$ ray spectra of all the sources studied here are better described by the LP model than the PL model. In this work, the $\gamma$-ray spectra of all the sources at all the epochs are better described by the LP model except for epoch B of 3C 273, which is well fit by the PL model. The parameters $\alpha$ and $\beta$ in the LP model fits to the data carry very important information on the characteristics of the $\gamma$-ray spectra. In this model, $\alpha$ gives the slope of the spectra and $\beta$ is a measure of the curvature in the spectra. A smaller value of $\alpha$ and $\beta$ implies a harder spectrum with a mild curvature. Any changes in the value of $\alpha$ and $\beta$ during different epochs is a measure of the changes in the $\gamma$-ray spectral shape. The dependence of $\alpha$ and $\beta$ values against the fluxes of the sources are given in Fig. 17. For all the sources we found the spectra to harden with increasing flux. We found decreasing as well as increasing trend of $\beta$ with flux. The variation in the $\gamma$-ray spectral shape can be associated with the shift in IC peak frequency. This is evident from the results of our SED analysis. Our model fits to the observed SED also gives the IC peak frequency (see Table 5). Analysis of the IC peak indicates that as the IC peak shifts towards lower energies, the spectrum is harder and the curvature $(\beta)$ is sharper which too demonstrates that the $\gamma$-ray spectral variation is closely related to the changes in the IC peak. Alternatively, $\gamma$-ray spectral variation can also be attributed to the changes in the location of the $\gamma$-ray emission region during different activity states of the sources (Coogan et al. 2016). Besides, since the $\gamma$-ray emission in FSRQs is due to EC scattering of the external target photons, the $\gamma$-ray peak energy will depend on the dominant external photon frequency. If the target photon field is the IR emission from the dusty torus, then the temperature of the dust emission will depend on the location of the emission region from the central black hole (Dermer et al. 2014; Ghisellini \& Tavecchio 2009).

\subsection{Connection between optical and $\mathrm{GeV}$ flux variation}

The capability of Fermi to scan the sky once in three hours and supporting ground based monitoring observations in the optical band has enabled one to study close correlations between flux variations in the $\mathrm{GeV}$ band and other low energy bands. From multiband observations of the blazar 3C 454.3 over a period of about 5 months, Bonning et al. (2009) found close correlation between the optical and GeV band flux variations. This argues for co-spatiality of the optical and GeV emission regions. This correlation is also easily understood in the one zone leptonic emission model, wherein relativistic electrons in the jet produce optical emission by synchrotron process, and the same relativistic electrons produce $\gamma$-ray emission by inverse Compton process. However, analysis of the same source by Rajput et al. (2019) 
noticed that the optical and $\mathrm{GeV}$ flux variations are not correlated at all times. Such mismatch between optical and $\mathrm{GeV}$ flux variations are also known in few other blazars such as PKS 0208-512, (Chatterjee et al. 2013), PKS 0454-234, S4 1849+67, BZQ J0850-1213, OP 313 (Cohen et al. 2014), PKS 2142-75 (Dutka et al. 2013), PKS 1510-089 (MacDonald et al. 2015) and PKS 2155-304 (Wierzcholska et al. 2019). From the analysis of multi-band light curves of the sources, we found instances where the optical and $\gamma$-ray flux variations are closely correlated, cases where there are optical flares without $\gamma$-ray counterpart and instances when there are $\gamma$-ray flares without optical counterparts. Thus, it is evident that the correlations between the optical and $\mathrm{GeV}$ flux variations in Fermi blazars are complex. Recently, from correlation analysis between the optical and $\gamma$-ray light curves of 178 blazars, Liodakis et al. (2019) found that statistically about $50 \%$ of their optical flares have no GeV counterparts and this fraction is less in the case of $\gamma$-ray flares, i.e., about $20 \%$ of $\gamma$-ray flares have no optical counterparts. While in the leptonic scenario a close correlation between optical and $\mathrm{GeV}$ variations is expected, the results found in this work as well as the other recent results by Rajput et al. (2019) and Liodakis et al. (2019) indicate that correlated variability analysis between the optical and $\mathrm{GeV}$ bands may also not be definitive in constraining the leptonic v/s hadronic scenario for the high energy emission process in blazars. Most of the correlation studies between different energy bands of the blazars indicate positive correlation. But there are exceptions and cases of anticorrelation are also found for some sources (Chatterjee et al. 2013; Cohen et al. 2014; Dutka et al. 2013; MacDonald et al. 2015; Rajput et al. 2019). We found various behaviours between optical and $\gamma$-ray energy bands for our selected sample of sources. We looked for a correlation between the optical (V-band) and $\gamma$-rays for all the epochs considered here. Fig. 18 shows only the epochs where the correlation is significant at the $90 \%$ level. We converted the $\gamma$-ray fluxes from ph $\mathrm{cm}^{-2} \mathrm{~s}^{-1}$ to erg $\mathrm{cm}^{-2} \mathrm{~s}^{-1}$ at $100 \mathrm{MeV}$ (Singal et al. 2014) to match the optical flux units. The results of the fit are given in Table 7. The fit takes into account the uncertainty in both optical and $\gamma$-rays. For the source PKS 1510-089 during epochs B and F the optical and $\gamma$-ray flares are correlated. During this epoch $\Gamma$ is larger than that of the quiescent period. This has given rise to increased flare in optical and $\gamma$-rays. The difference in the amplitude of variations between optical and $\gamma$-ray flares during epochs $\mathrm{B}$ and $\mathrm{F}$ must be due to a combination of $\Gamma$ and $\mathrm{B}$. For epochs $\mathrm{A}, \mathrm{C}$ and $\mathrm{E}$ the magnetic field is lower than the quiescent period by a factor of 1.2 - 1.5. This has led to decreased optical variations. At the same time $\Gamma$ has increased from $1.1-1.7$ times the quiescent period leading to increased $\gamma$-ray flare, but no corresponding optical flare (see Fig. 2 and Fig. 3).

In the source $3 \mathrm{C} 273$, using our criteria, we were able to identify one quiescent period and one flaring period. At epoch A, the bulk Lorentz factor increased compared to the quiescent state B, whether the magnetic field is nearly the same (Table 5). It is natural to expect increased optical and $\gamma$-ray flares during epoch A, but we found a $\gamma$-ray flare without an optical counterpart. This absence in optical flux variations might be due to sub-dominant optical synchrotron emission compared to the prominent accretion disk emission. The prominent accretion disk component is very well evident in the broad band SED both in the quiescent and active states (Fig. 14).

In the source 3 C 279, we identified four epochs, A,B, C and D of which during epoch $\mathrm{A}$, the source was quiescent while it was active during the other epochs. During epoch D, magnetic field is about 1.5 times larger than the quiescent period leading to larger optical synchrotron emission. At the same epoch, $\Gamma$ has increased from about 7 to 12 . This explains the increased $\gamma$-ray and optical flaring in epoch D. During epochs B and C, $\Gamma$ has increased relative to the quiescent state giving rise to larger $\gamma$-ray fluctuations. During epoch $\mathrm{B}$, magnetic field is marginally larger than the quiescent period, while the particle density is lower than the quiescent period. However, in epoch $\mathrm{C}$, the magnetic field and particle density is lower and higher respectively than the quiescent period. The interplay between low magnetic field and high particle density and vice versa could lead to lower optical variations. This could be the reason for $\gamma$-ray flares without optical counterparts in epochs B and C in 3 C 279. The low energy peak of the SED during all epochs in 3C 279 is dominated by synchrotron emission from the relativistic jet.

In CTA 102, we found one flaring epoch when the optical and $\gamma$-ray seems to be correlated. Many short term flares with optical and $\gamma$-ray counterparts are seen during this epoch. For SED analysis we considered only 10 days due to the availability of $\gamma$-ray, X-ray and optical data points for SED modelling. During Epoch B, $\Gamma$ was nearly four times greater than the quiescent epoch. The magnetic field during epoch B and the quiescent period agree with each other within errors (see Table 5). This increase in $\Gamma$ relative to the quiescent epoch is the cause of the increased $\gamma$-ray flare and optical flare during epoch B. Prominent accretion disk component is visible in the SED during the quiescent phase, however, this is not evident in the flaring epoch B (Fig. 16). This is also reflected in the high accretion rate found during the quiescent epoch $\mathrm{A}$ and a negligibly small accretion rate during epoch $\mathrm{B}$.

In the SED analysis carried out in this work, the viewing angle is fixed at $2^{\circ}$ for all the sources. Radio observations indicate that the average viewing angle of the sources studied here is lesser than $2^{\circ}$ except for 3C 273, where it is around $6^{\circ}$ (Jorstad et al. 2017). Similarly, according to Hovatta et al. (2009) the average viewing angle is around $3.5^{\circ}$ for the sources except CTA 102 for which it is $3.7^{\circ}$. Thus the values of viewing angle available in the literature of these source is generally low and not too different from the constant value of $2^{\circ}$ used for all the sources. However, to ascertain the effect viewing angle can have on the $\Gamma$ obtained from SED analysis, we repeated the SED analysis for different values of $\theta$ such as $0.5,1.0$, 1.5, 2.5 and 3.0 degrees. A similar fit statistics was obtained for all cases with considerable increase in the $\Gamma$ suggesting a degeneracy. The plot between $\Gamma \mathrm{v} / \mathrm{s} \theta$ for the source PKS $1510-089$ is shown in the Figure 19. We found that as $\theta$ increases, $\Gamma$ too increased. No significant changes were noticed in the other parameters, and the changes are consistent within the errors. The choice of viewing angle do not alter our conclusions since the Lorentz factor obtained for different epochs still follow the same trend. This trend is also found in other sources. To further verify the degeneracy between the bulk Lorentz factor and the viewing angle we continued the fitting procedure with the constant $\Gamma$, which is fixed at 15 and repeated the SED fitting for all the sources with the inclusion of the accretion disk component. The results of the SED fitting at constant $\Gamma$ are given in Table 6. We found minimal change in the parameters compared to their values given in Table 5. It shows that the adopted $\theta$ values directly impact the predicted $\Gamma$ or vice-versa and this is true for all the sources and at all epochs. Recent study from radio observations of the source S5 $0716+714$ by Kravchenko et al. (2020) also shows that the variation in $\Gamma$ occurs due to the change in $\theta$. This indicates that the changes in the $\gamma$-ray flux states of a source is largely associated with the changes in the $\Gamma$ of the jet, as well as $\theta$. Though various physical processes have been proposed to explain the uncorrelated optical and $\mathrm{GeV}$ flux variations in blazars in the literature, our analysis of the broad band SED of the four FSRQs studied in this work on different epochs is consistent with leptonic processes in the jets of these sources. 
Table 4. Values of the parameters that were frozen during the model fits to the observed SEDs. Here, $\mathrm{R}$ is the size of the emission region in units of $10^{15}$ $\mathrm{cm}$, and the temperature $\mathrm{T}$ is in Kelvin.

\begin{tabular}{lcrrrrc}
\hline Object & $\mathrm{R}$ & $\gamma_{\min }$ & $\gamma_{\max }$ & $\gamma_{b}$ & $\mathrm{~T}(\mathrm{~K})$ & $\mathrm{f}$ \\
\hline PKS 1510-089 & 7.9 & 40 & $2 \times 10^{4}$ & 1500 & 800 & 0.9 \\
3C 273 & 15.8 & 50 & $1 \times 10^{4}$ & 1200 & 800 & 0.9 \\
3C 279 & 15.8 & 40 & $2 \times 10^{4}$ & 1200 & 800 & 0.9 \\
CTA 102 & 100 & 50 & $2 \times 10^{4}$ & 2100 & 800 & 0.02 \\
\hline
\end{tabular}

\subsection{Optical spectral variations}

It is argued that FSRQs generally show a RWB trend (Sarkar et al. 2019), while BL Lacs show a BWB trend (Gaur et al. 2019). If these two classes of blazars indeed show a distinct colour magnitude relation, it leads to hypothesize that the jets are fundamentally different between FSRQs and BL Lacs. However, with the availability of more data it is now known that blazars show different types of colour variability in the optical - IR bands. Blazars are found to show BWB trend (e.g., Stalin et al. 2009), RWB trend (e.g., Sarkar et al. 2019), both BWB and RBW trends (Rajput et al. 2019) as well as weak/no spectral change with brightness (Raiteri et al. 2003). The dominance of the more variable red synchrotron emission over the less variable thermal emission from the accretion disk could lead to a RWB trend (Sarkar et al. 2019). Alternatively, a BWB trend can happen due to increased variations at shorter wavelengths (Stalin et al. 2009). In the one zone leptonic emission model this can be explained via the injection of fresh electrons with high energy leading to a BWB trend (Kirk et al. 1998; Mastichiadis \& Kirk 2002). Alternatively, according to Papadakis et al. (2007) and Villata et al. (2004) a BWB trend can also happen due to changes in the Doppler factor. We examined the colour variations during all the epochs and considered a source to show colour variation only when the Spearman rank correlation coefficient is $>0.5$ as well as $<-0.5$ with the null hypothesis probability $<0.05$. The observed colour variation is significant at the $95 \%$ level. In the case of PKS 1510-089 significant colour variations were observed during epoch A,E and F. While during epochs A and E, we found a RWB trend, during epoch R, we found a BWB trend. The observed colour variation in the optical band has no direct relation to the presence or absence of correlated variation between optical and $\gamma$-ray bands. Our results clearly indicate that FSRQs show both BWB and RWB behaviours.

\section{SUMMARY}

We carried out detailed investigations of the correlation between optical and GeV flux variations in four FSRQs namely PKS 1510-089, 3C 273, 3C 279 and CTA 102. Our study includes (a) identification of epochs in those sources with anomalous optical-GeV flux variations, (b) analysis of the broad band SEDs of the sources in those epochs, (c) the analysis of $\gamma$-ray spectra and (d) analysis of optical-IR colour variations. The results of those analysis are summarized below:

(i) All the four FSRQs studied here, namely PKS 1510-089, 3C 279, 3C 273 and CTA 102 showed varied correlations between optical and $\mathrm{GeV}$ flux variations. We found cases when (a) the optical and $\gamma$-rays are closely correlated, (b) epochs when there are optical flares without $\gamma$-ray and (c) epochs when there are $\gamma$-ray flares without optical counterparts. From our one zone leptonic model fit to the observed SED of all such epochs in the sources, we found that the regions giving rise to optical and $\gamma$-ray flares are co-spatial.

(ii) SED analysis indicates that the optical emission is often well explained by synchrotron emission process and the $\gamma$-ray emission is well explained by EC process with the seed photons from the torus. A Prominent accretion disk component is seen in the synchrotron part of the SEDs in PKS 1510-089, 3C 273 and the quiescent state SED of CTA 102. For 3C279, there is no evidence of an accretion disk component in the synchrotron part of the SED.

(iii) From model fits we found that (a) correlated optical and $\gamma$-ray flux variations are caused by increase in the bulk Lorentz factor (b) $\gamma$-ray flares with no optical counterparts are due to an increase in the bulk Lorentz factor and/or increase in the electron number density and (c) an optical flare with no $\gamma$-ray counterpart is due to an increase in the magnetic field.

(iv) The $\gamma$-ray spectra of the sources during various epochs are well represented by the LP model

(v) Varied colour behaviours such as BWB trend and RWB trend are seen in our sample of sources.

\section{ACKNOWLEDGMENTS}

We thank the referee for his/her comments that helped the authors to improve the manuscript. This paper has made use of upto-date SMARTS optical/near infra-red light curves that are available at www.astro.yale.edu/smarts/glast/home.php. Also, data from the Steward Observatory spectropolarimetric monitoring project were used. This program is supported by Fermi Guest Investigator grants NNX08AW56G, NNX09AU10G, NNX12AO93G, and NNX15AU81G. This research has made use of the High Performance Computing Facility of the Indian Institute of Astrophysics, Bangalore.

\section{DATA AVAILABILITY}

The multiwavelength data underlying this article are publicly available from the Fermi-LAT ${ }^{7}$, Swift-XRT and Swift-UVOT ${ }^{8}$, SMARTS $^{9}$ and Steward observatory ${ }^{10}$.

\section{REFERENCES}

Abdo A. A., et al., 2009, ApJ, 700, 597

Abdo A. A., et al., 2010a, Nature, 463, 919

Abdo A. A., et al., 2010b, ApJ, 710, 1271

Abdo A. A., et al., 2010c, ApJ, 714, L73

Abdo A. A., et al., 2010d, ApJ, 716, 30

Abdo A. A., et al., 2010e, ApJ, 721, 1425

Ackermann M., et al., 2015, ApJ, 810, 14

Aharonian F. A., 2000, New Astron., 5, 377

Aleksić J., et al., 2014, A\&A, 569, A46

Andruchow I., Romero G. E., Cellone S. A., 2005, A\&A, 442, 97

Angel J. R. P., Stockman H. S., 1980, ARA\&A, 18, 321

Antonucci R., 1993, ARA\&A, 31, 473

Arnaud K. A., 1996, in Jacoby G. H., Barnes J., eds, Astronomical Society of the Pacific Conference Series Vol. 101, Astronomical Data Analysis Software and Systems V. p. 17

Atwood W. B., et al., 2009, ApJ, 697, 1071

Bastieri D., 2009, The Astronomer's Telegram, 2168, 1

Begelman M. C., et al., 1987, ApJ, 322, 650

\footnotetext{
7 https://fermi.gsfc.nasa.gov/ssc/data/access/

8 https://www.ssdc.asi.it/mmia/index.php?mission=swiftmastr

9 http://www.astro.yale.edu/smarts/glast/home.php

$10 \mathrm{http} / / /$ james.as.arizona.edu/ psmith/Fermi/DATA/individual.html
} 

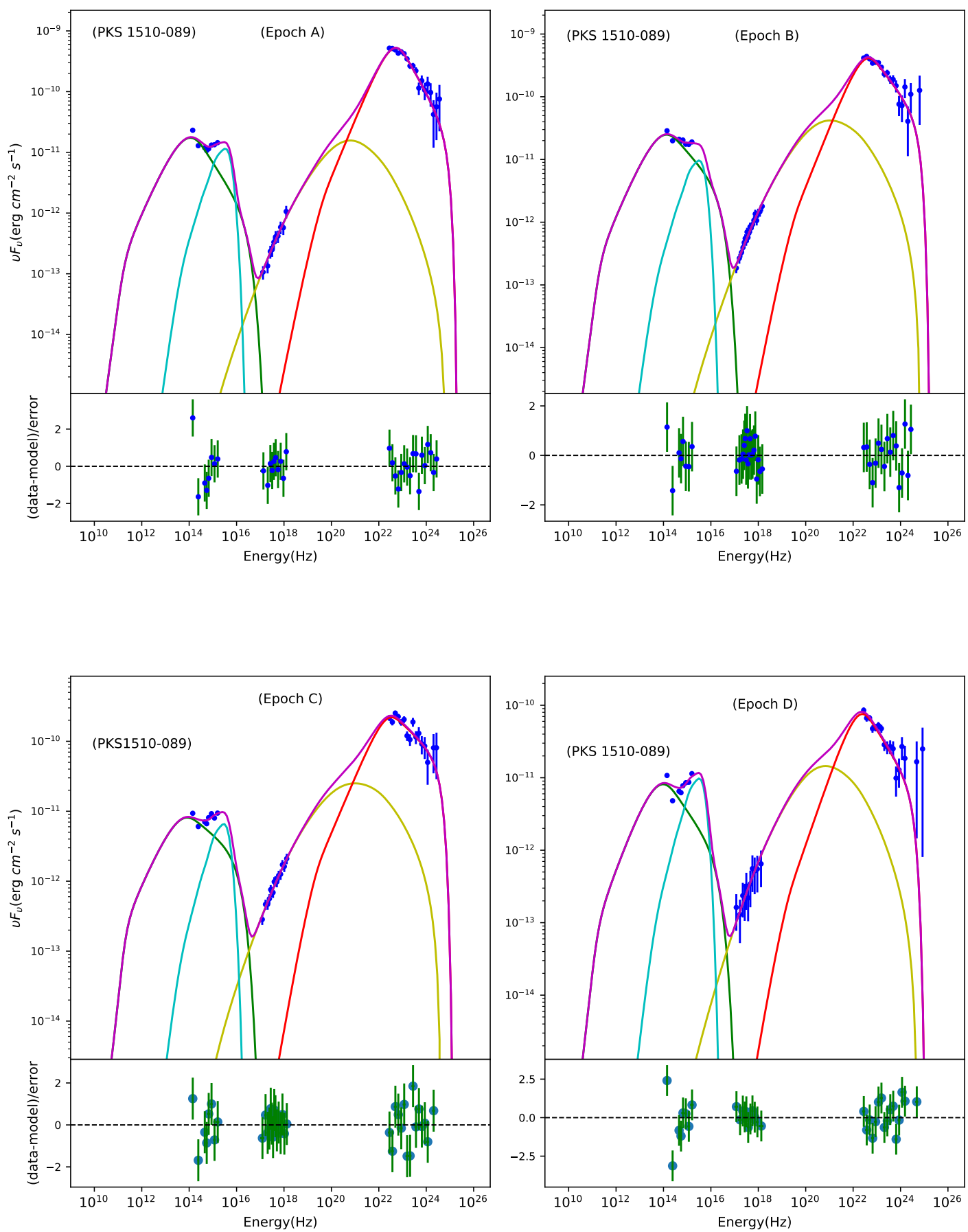

Figure 12. Observed broad band spectral energy distribution for the source PKS 1510-089 along with model fits for the epochs A,B C and D. The various components are the synchrotron emission (green line), the SSC process (yellow line), the EC process (red line) and the accretion disk component (cyan line). The magenta line is the sum of all the components. The bottom panel in each SED residuals obtained by model fits to the observed data points obtained from XSPEC.

Błażejowski M., Sikora M., Moderski R., Madejski G. M., 2000, ApJ, 545, 107

Boettcher M., Mause H., Schlickeiser R., 1997, A\&A, 324, 395

Bolton J. G., Ekers J., 1966, Australian Journal of Physics, 19, 559

Bonning E. W., et al., 2009, ApJ, 697, L81

Bonning E., et al., 2012, ApJ, 756, 13

Böttcher M., 2007, Ap\&SS, 309, 95

Böttcher M., Reimer A., Sweeney K., Prakash A., 2013, ApJ, 768, 54
Breeveld A. A., Landsman W., Holland S. T., Roming P., Kuin N. P. M., Page M. J., 2011, in McEnery J. E., Racusin J. L., Gehrels N., eds, American Institute of Physics Conference Series Vol. 1358, American Institute of Physics Conference Series. pp 373-376 (arXiv : 1102 .4717), doi:10.1063/1.3621807

Burrows D. N., et al., 2005, Space Sci. Rev., 120, 165

Carnerero M. I., et al., 2015, MNRAS, 450, 2677

Cerruti M., Dermer C. D., Lott B., Boisson C., Zech A., 2013, ApJ, 771, L4 

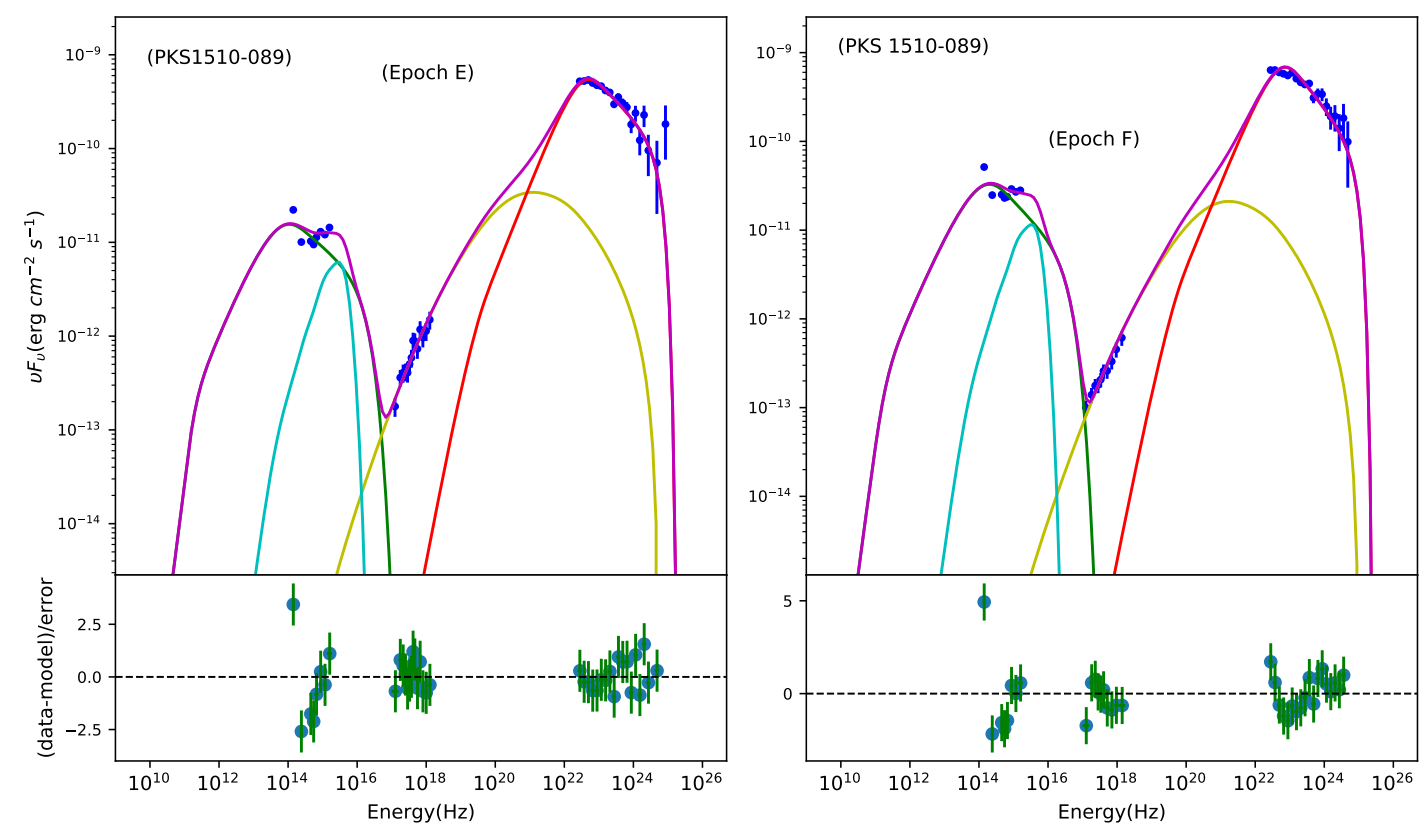

Figure 13. Model fits to the broad band SED during epochs E and F for the source PKS 1510-089. The lines and symbols are as in Fig. 12
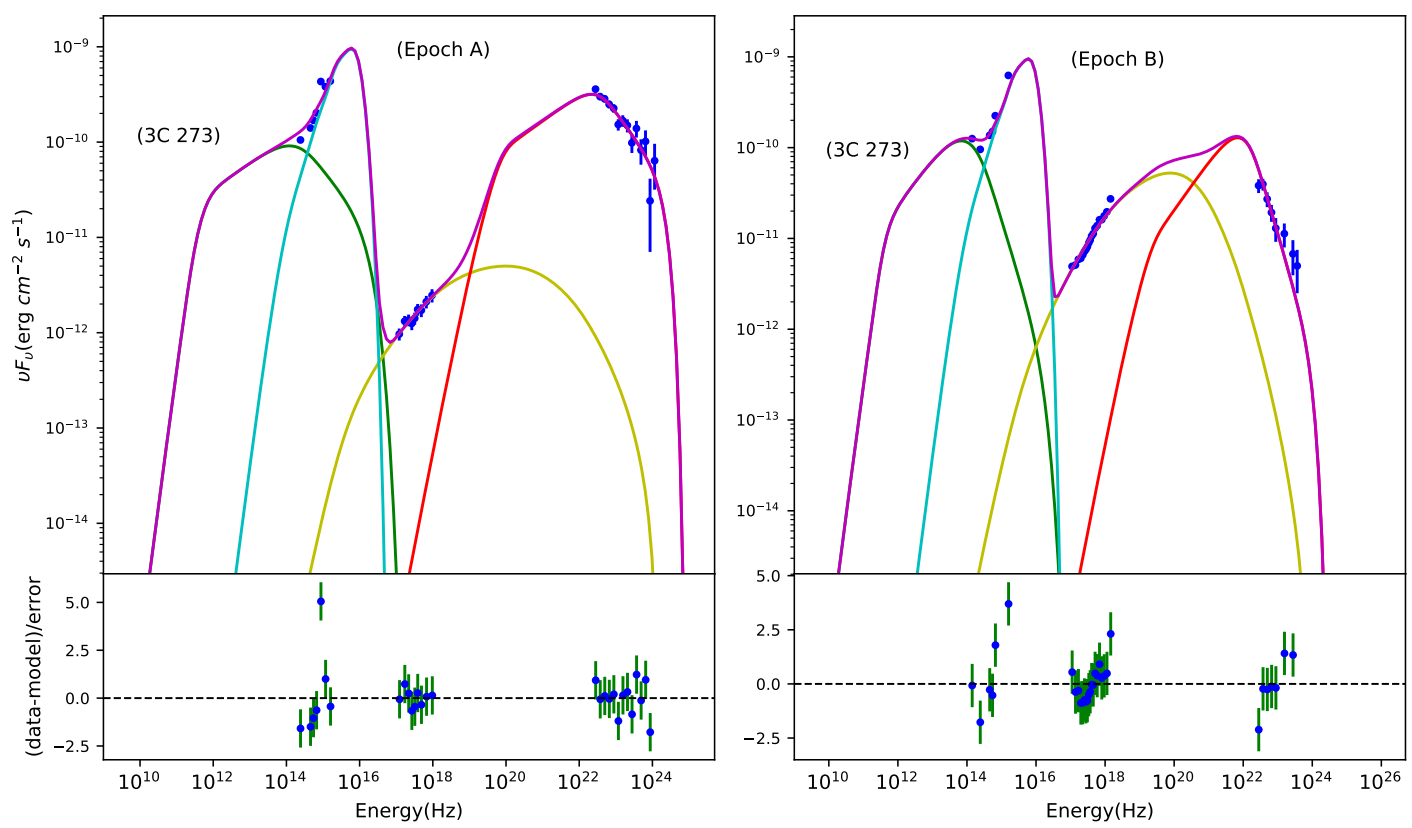

Figure 14. Model fits to the broad band SED during epochs A and B for source 3C 273. The lines and symbols are as in Fig. 12

Chatterjee R., et al., 2008, ApJ, 689, 79

Chatterjee R., et al., 2012, ApJ, 749, 191

Chatterjee R., et al., 2013, ApJ, 763, L11

Chen L., 2018, ApJS, 235, 39

Ciprini S., 2016, The Astronomer's Telegram, 9869

Cohen D. P., Romani R. W., Filippenko A. V., Cenko S. B., Lott B., Zheng W., Li W., 2014, ApJ, 797, 137

Coogan R. T., Brown A. M., Chadwick P. M., 2016, MNRAS, 458, 354

Costamante L., Cutini S., Tosti G., Antolini E., Tramacere A., 2018, MNRAS, 477,4749
Dermer C. D., Schlickeiser R., 1993, ApJ, 416, 458

Dermer C. D., Cerruti M., Lott B., Boisson C., Zech A., 2014, ApJ, 782, 82 Diltz C., Böttcher M., 2016, ApJ, 826, 54

Dutka M. S., et al., 2013, ApJ, 779, 174

Fichtel C. E., et al., 1994, ApJS, 94, 551

Fossati G., Maraschi L., Celotti A., Comastri A., Ghisellini G., 1998, MNRAS, 299, 433

Gaur H., et al., 2019, MNRAS, 484, 5633

Gehrels N., et al., 2004, ApJ, 611, 1005

Ghisellini G., Madau P., 1996, MNRAS, 280, 67 

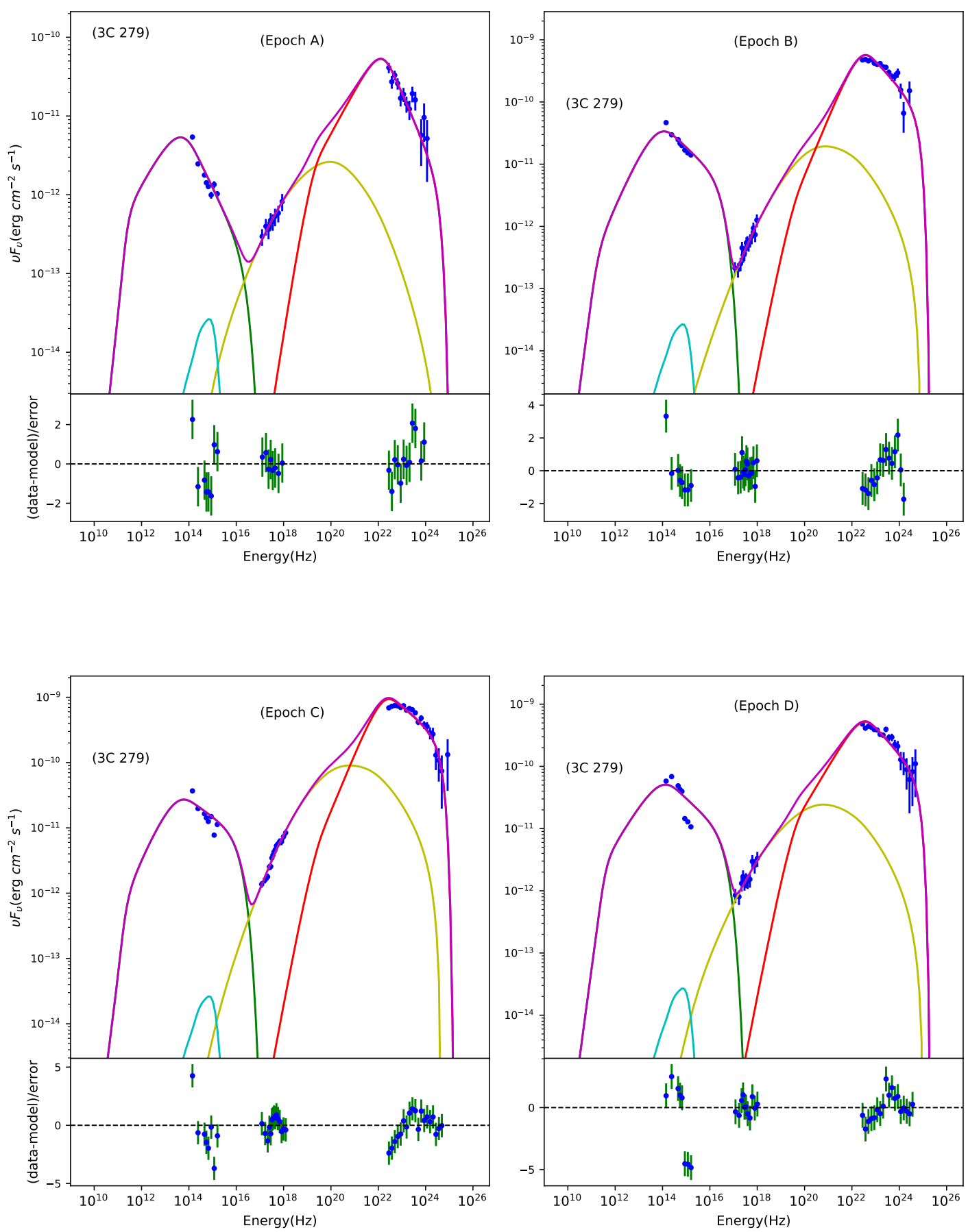

Figure 15. Model fits to the broad band SED during epochs A, B, C and D for the source 3C 279. The lines and symbols are as in Fig. 12

Ghisellini G., Maraschi L., 1989, ApJ, 340, 181

Ghisellini G., Tavecchio F., 2008, MNRAS, 387, 1669

Ghisellini G., Tavecchio F., 2009, MNRAS, 397, 985

Ghisellini G., Tavecchio F., Foschini L., Ghirland a G., 2011, MNRAS, 414, 2674

H. E. S. S. Collaboration et al., 2013, A\&A, 554, A107

Harris D. E., Krawczynski H., 2006, ARA\&A, 44, 463

Harris J., Daniel M. K., Chadwick P. M., 2012, ApJ, 761, 2

Harris J., Chadwick P. M., Daniel M. K., 2014, MNRAS, 441, 3591

Hartman R. C., et al., 1992, ApJ, 385, L1

Hartman R. C., et al., 1999, ApJS, 123, 79
Hayashida M., Madejski G., Blandford R., Asano K., Larsson S., FermiLAT Collaboration Nalewajko K., Sikora M., 2017, in 6th International Symposium on High Energy Gamma-Ray Astronomy. p. 050015, doi:10.1063/1.4968961

Hovatta T., Valtaoja E., Tornikoski M., Lähteenmäki A., 2009, A\&A, 494, 527

IceCube Collaboration et al., 2018, Science, 361, 147

Jolley E. J. D., Kuncic Z., 2008, ApJ, 676, 351

Jorstad S. G., et al., 2017, ApJ, 846, 98

Kalberla P. M. W., Burton W. B., Hartmann D., Arnal E. M., Bajaja E., Morras R., Pöppel W. G. L., 2005, A\&A, 440, 775 

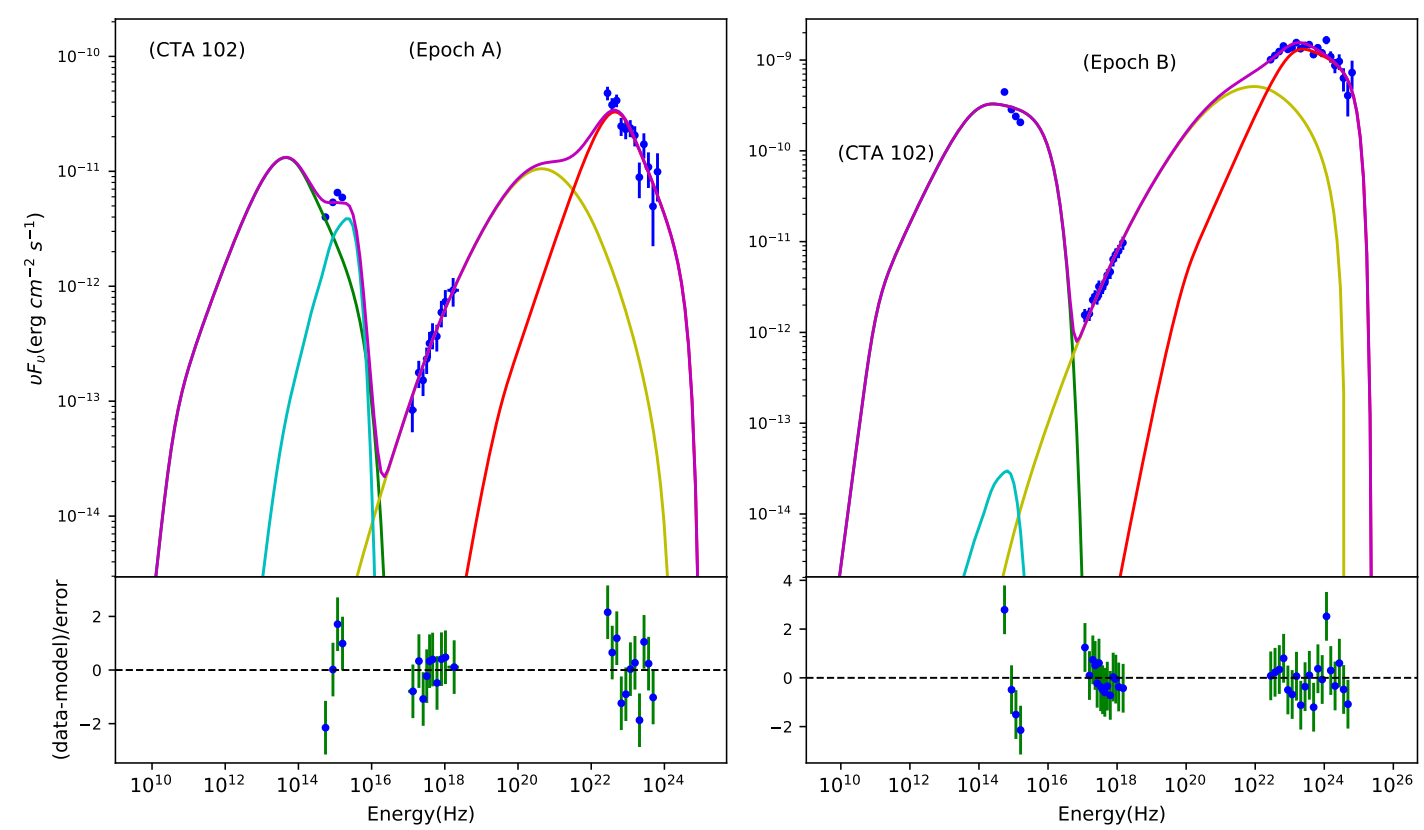

Figure 16. Model fits to the broad band SED during epoch A and B for the source CTA 102. The lines and symbols are as in Fig. 12

Table 5. Results of the broad band SED analysis of the sources at different epochs for the constant viewing angle $\theta=2^{\circ}$

\begin{tabular}{|c|c|c|c|c|c|c|c|c|c|}
\hline Name & Epoch & $\begin{array}{r}\text { Bulk Lorentz } \\
\text { factor }\end{array}$ & $\begin{array}{c}\text { Low energy } \\
\text { particle index }\end{array}$ & $\begin{array}{c}\text { High energy } \\
\text { particle index }\end{array}$ & $\begin{array}{l}\text { Eletron energy } \\
\text { density }\left(\mathrm{cm}^{-3}\right)\end{array}$ & $\begin{array}{c}\text { Magnetic } \\
\text { field (Gauss) }\end{array}$ & $\begin{array}{r}\text { Accretion } \\
\text { rate } \\
\end{array}$ & $\begin{array}{c}\text { IC peak } \\
(\mathrm{MeV})\end{array}$ & $\chi^{2} /$ dof \\
\hline \multirow{8}{*}{ PKS 1510-089 } & A (with AD) & $10.62 \pm 1.20$ & $1.10 \pm 0.58$ & $4.22 \pm 0.16$ & $0.12 \pm 0.04$ & $0.84 \pm 0.07$ & $1.48 \pm 0.32$ & & 0.8 \\
\hline & $\mathrm{B}$ (with AD) & $9.01 \pm 0.69$ & $1.10 \pm 0.38$ & $3.86 \pm 0.12$ & $0.20 \pm 0.05$ & $0.98 \pm 0.06$ & $1.24 \pm 0.48$ & & 0.5 \\
\hline & $\mathrm{C}$ (without $\mathrm{AD}$ ) & $5.72 \pm 0.61$ & $1.10 \pm 0.35$ & $3.04 \pm 0.13$ & $0.66 \pm 0.19$ & $0.66 \pm 0.03$ & - & 163 & 1.2 \\
\hline & $\mathrm{C}$ (with $\mathrm{AD})$ & $7.31 \pm 0.63$ & $1.39 \pm 0.23$ & $3.55 \pm 0.13$ & $0.37 \pm 0.09$ & $0.64 \pm 0.05$ & $0.85 \pm 0.22$ & & 0.7 \\
\hline & $\mathrm{E}$ (without $\mathrm{AD}$ ) & $8.62 \pm 0.86$ & $1.10 \pm 0.51$ & $3.45 \pm 0.08$ & $0.27 \pm 0.09$ & $0.72 \pm 0.06$ & - & 209 & 1.6 \\
\hline & $\mathrm{E}$ (with $\mathrm{AD}$ ) & $9.22 \pm 0.96$ & $1.13 \pm 0.27$ & $3.63 \pm 0.10$ & $0.24 \pm 0.08$ & $0.68 \pm 0.06$ & $0.79 \pm 0.28$ & & 1.3 \\
\hline & $\mathrm{F}$ (without $\mathrm{AD}$ ) & $12.31 \pm 1.19$ & $1.10 \pm 0.37$ & $3.68 \pm 0.09$ & $0.08 \pm 0.02$ & $1.18 \pm 0.07$ & - & 333 & 2.2 \\
\hline & $\mathrm{F}$ (with $\mathrm{AD})$ & $12.53 \pm 1.11$ & $1.10 \pm 0.33$ & $3.81 \pm 0.12$ & $0.07 \pm 0.02$ & $1.12 \pm 0.06$ & $1.49 \pm 0.67$ & & 1.9 \\
\hline \multirow[t]{2}{*}{$3 \mathrm{C} 273$} & A (with AD) & $9.42 \pm 1.37$ & $2.44 \pm 0.27$ & $3.94 \pm 0.15$ & $0.01 \pm 0.004$ & $2.21 \pm 0.42$ & $16.00 \pm 4.13$ & 121 & 1.9 \\
\hline & B (with AD) & $5.00 \pm 0.52$ & $1.92 \pm 0.12$ & $5.48 \pm 0.44$ & $0.07 \pm 0.009$ & $2.33 \pm 0.20$ & $16.00 \pm 2.72$ & 32 & 1.5 \\
\hline \multirow{4}{*}{ 3C 279} & $\mathrm{C}$ (without $\mathrm{AD}$ ) & $8.80 \pm 0.04$ & $1.24 \pm 0.20$ & $3.51 \pm 0.07$ & $0.22 \pm 0.03$ & $0.66 \pm 0.04$ & - & 147 & 1.8 \\
\hline & $\mathrm{C}$ (with $\mathrm{AD}$ ) & $8.80 \pm 0.60$ & $1.24 \pm 0.21$ & $3.51 \pm 0.09$ & $0.22 \pm 0.04$ & $0.66 \pm 0.04$ & $0.01 \pm 1.86$ & & 1.9 \\
\hline & $\mathrm{D}$ (without $\mathrm{AD}$ ) & $11.75 \pm 1.53$ & $1.72 \pm 0.25$ & $3.70 \pm 0.01$ & $0.05 \pm 0.01$ & $1.52 \pm 0.13$ & - & 154 & 3.0 \\
\hline & $\mathrm{D}$ (with $\mathrm{AD}$ ) & $11.75 \pm 1.50$ & $1.72 \pm 0.26$ & $3.70 \pm 0.11$ & $0.05 \pm 0.01$ & $1.52 \pm 0.18$ & $0.01 \pm 4.92$ & & 3.1 \\
\hline \multirow[t]{4}{*}{ CTA 102} & $\mathrm{~A}$ (without AD) & $7.98 \pm 1.50$ & $1.10 \pm 0.64$ & $3.84 \pm 0.46$ & $0.006 \pm 0.004$ & $0.39 \pm 0.10$ & - & 196 & 2.4 \\
\hline & A (with AD) & $8.42 \pm 1.19$ & $1.10 \pm 0.70$ & $4.35 \pm 0.38$ & $0.006 \pm 0.003$ & $0.32 \pm 0.07$ & $10.00 \pm 6.09$ & & 1.4 \\
\hline & $\mathrm{B}$ (without AD) & $32.17 \pm 13.61$ & $1.29 \pm 0.08$ & $3.17 \pm 0.15$ & $0.007 \pm 0.001$ & $0.42 \pm 0.03$ & - & 1295 & 1.0 \\
\hline & B (with AD) & $36.44 \pm 11.84$ & $1.28 \pm 0.09$ & $3.11 \pm 0.17$ & $0.008 \pm 0.003$ & $0.42 \pm 0.10$ & $0.08 \pm 325.54$ & & 1.0 \\
\hline
\end{tabular}

Kaur N., Baliyan K. S., 2018, A\&A, 617, A59

Kinman T. D., Lamla E., Wirtanen C. A., 1966, ApJ, 146, 964

Kirk J. G., Rieger F. M., Mastichiadis A., 1998, A\&A, 333, 452

Konigl A., 1981, ApJ, 243, 700

Kravchenko E. V., et al., 2020, ApJ, 893, 68

Liao N. H., Bai J. M., Liu H. T., Weng S. S., Chen L., Li F., 2014, ApJ, 783,
83

Liodakis I., Romani R. W., Filippenko A. V., Kocevski D., Zheng W., 2019, ApJ, 880, 32

Lynden-Bell D., 1969, Nature, 223, 690

Lynds C. R., Stockton A. N., Livingston W. C., 1965, ApJ, 142, 1667

MAGIC Collaboration et al., 2008, Science, 320, 1752 
Table 6. Results of the broad band SED analysis on the sources at different epochs for the constant Bulk Lorentz factor $\Gamma=15$.

\begin{tabular}{llrcccccc}
\hline Name & Epoch & $\begin{array}{r}\text { Viewing } \\
\text { Angle }\end{array}$ & $\begin{array}{c}\text { Low energy } \\
\text { particle index }\end{array}$ & $\begin{array}{c}\text { High energy } \\
\text { particle index }\end{array}$ & $\begin{array}{c}\text { Eletron energy } \\
\text { density }\left(\mathrm{cm}^{-3}\right)\end{array}$ & $\begin{array}{c}\text { Magnetic } \\
\text { field (Gauss) }\end{array}$ & $\begin{array}{r}\text { Accretion } \\
\text { rate }\end{array}$ & $\chi^{2} /$ dof \\
\hline PKS 1510-089 & A & $2.97 \pm 0.34$ & $1.10 \pm 0.57$ & $4.24 \pm 0.16$ & $0.12 \pm 0.04$ & $0.84 \pm 0.07$ & $1.50 \pm 0.31$ & 0.8 \\
& B & $3.46 \pm 0.24$ & $1.12 \pm 0.19$ & $3.87 \pm 0.12$ & $0.19 \pm 0.05$ & $0.99 \pm 0.06$ & $1.25 \pm 0.48$ & 0.5 \\
& C & $4.17 \pm 0.29$ & $1.39 \pm 0.23$ & $3.55 \pm 0.13$ & $0.37 \pm 0.09$ & $0.64 \pm 0.05$ & $0.85 \pm 0.22$ & 0.7 \\
& D & $4.63 \pm 0.70$ & $1.10 \pm 1.26$ & $3.95 \pm 0.18$ & $0.21 \pm 0.15$ & $0.98 \pm 0.16$ & $1.26 \pm 0.22$ & 1.2 \\
& E & $3.41 \pm 0.33$ & $1.14 \pm 0.27$ & $3.63 \pm 0.10$ & $0.24 \pm 0.08$ & $0.68 \pm 0.06$ & $0.79 \pm 0.28$ & 1.3 \\
& F & $2.50 \pm 0.25$ & $1.10 \pm 0.33$ & $3.82 \pm 0.12$ & $0.07 \pm 0.02$ & $1.12 \pm 0.06$ & $1.50 \pm 0.67$ & 1.9 \\
\hline 3C 273 & A & $3.17 \pm 0.46$ & $2.56 \pm 0.27$ & $4.00 \pm 0.16$ & $0.01 \pm 0.003$ & $2.21 \pm 0.40$ & $16.00 \pm 3.69$ & 1.9 \\
& B & $6.26 \pm 0.29$ & $1.65 \pm 0.12$ & $4.80 \pm 0.40$ & $0.11 \pm 0.02$ & $2.04 \pm 0.16$ & $16.00 \pm 2.73$ & 1.1 \\
\hline 3C 279 & A & $4.14 \pm 0.52$ & $2.03 \pm 0.46$ & $4.24 \pm 0.19$ & $0.07 \pm 0.02$ & $1.08 \pm 0.15$ & $0.01 \pm 0.22$ & 1.7 \\
& B & $2.55 \pm 0.28$ & $1.36 \pm 0.21$ & $3.73 \pm 0.11$ & $0.03 \pm 0.01$ & $1.26 \pm 0.09$ & $0.01 \pm 2.65$ & 1.3 \\
& C & $3.56 \pm 0.22$ & $1.24 \pm 0.21$ & $3.51 \pm 0.09$ & $0.22 \pm 0.04$ & $0.66 \pm 0.04$ & $0.01 \pm 1.80$ & 1.9 \\
& D & $2.68 \pm 0.38$ & $1.72 \pm 0.26$ & $3.70 \pm 0.11$ & $0.05 \pm 0.01$ & $1.52 \pm 0.17$ & $0.01 \pm 5.90$ & 3.1 \\
\hline CTA 102 & A & $3.71 \pm 0.45$ & $1.10 \pm 0.70$ & $4.37 \pm 0.38$ & $0.006 \pm 0.003$ & $0.32 \pm 0.07$ & $10.00 \pm 5.86$ & 1.4 \\
& B & $1.38 \pm 0.49$ & $1.25 \pm 0.11$ & $2.98 \pm 0.18$ & $0.009 \pm 0.004$ & $0.40 \pm 0.12$ & $0.25 \pm 388.08$ & 1.1 \\
\hline
\end{tabular}
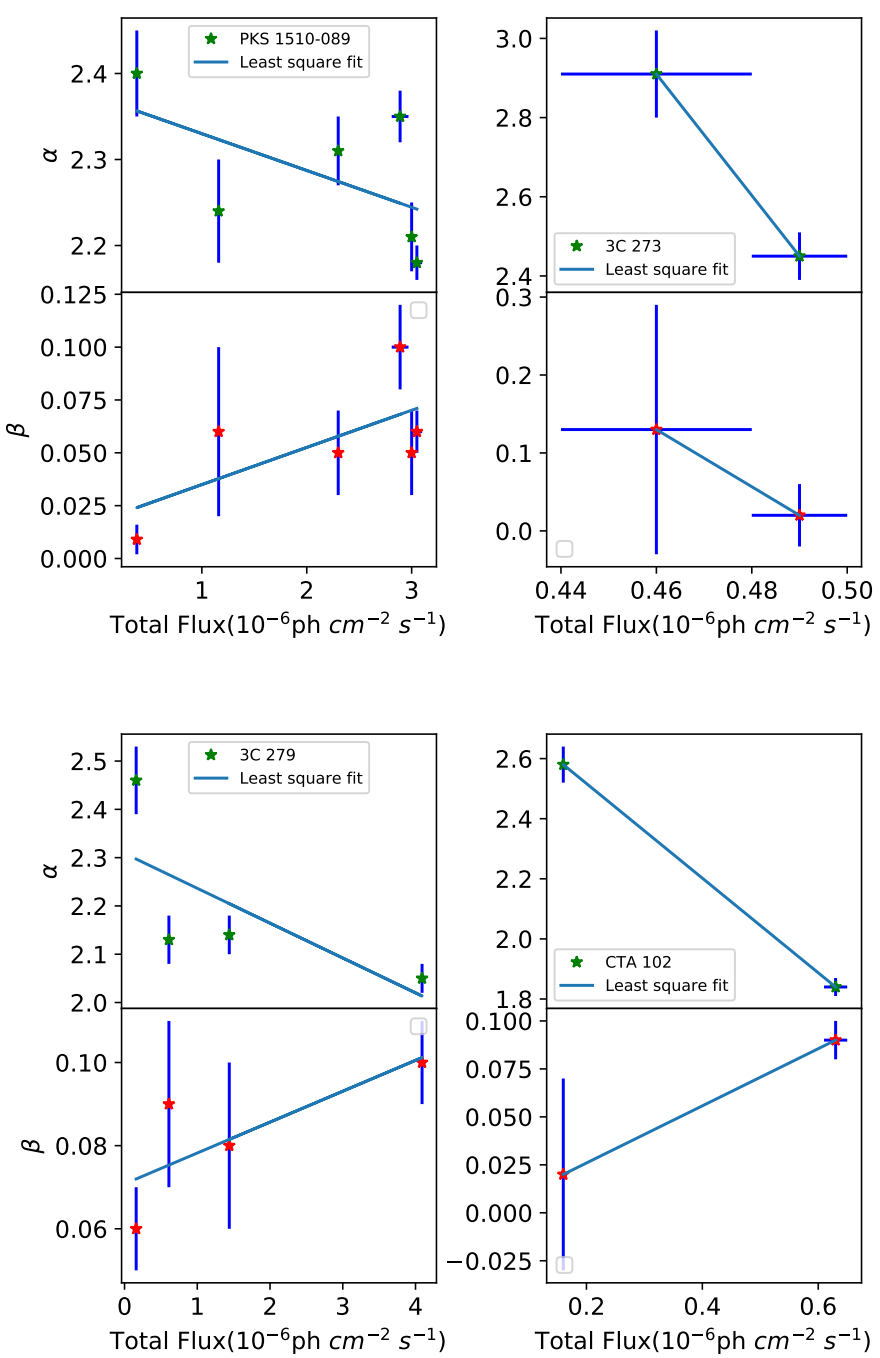

Figure 17. Variations of the parameters $\alpha$ and $\beta$ with flux for the sources PKS 1510-089 (top left), 3C 273 (top right), 3C 279 (bottom left) and CTA 102 (bottom right).
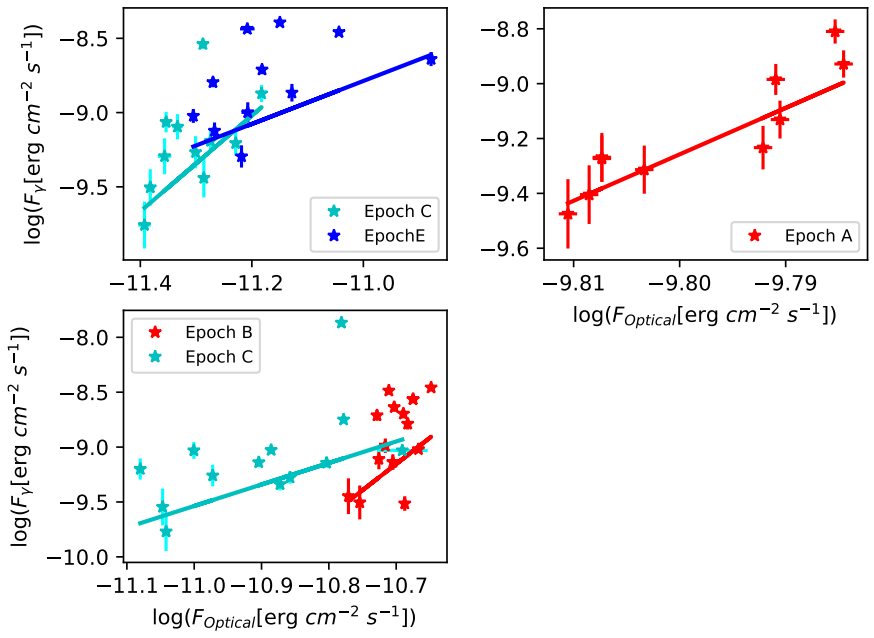

Figure 18. Optical flux v/s $\gamma$-ray flux for the sources PKS 1510-089 (top left), 3C 273 (top right) and 3C 279 (bottom left) respectively.

Table 7. Results of the linear least squares fit to the optical and $\gamma$-ray flux measurements, during different epochs for the sources PKS 1510-089, 3C 273, and 3C 279. Here R and P are the Spearman rank correlation coefficient and the probability for no correlation respectively.

\begin{tabular}{lcrrcc}
\hline Object & Epoch & Slope & Intercept & $\mathrm{R}$ & $\mathrm{P}$ \\
\hline PKS 1510-089 & $\mathrm{C}$ & $3.23 \pm 1.07$ & $27.11 \pm 12.09$ & 0.55 & 0.08 \\
& $\mathrm{E}$ & $1.45 \pm 0.60$ & $7.13 \pm 6.73$ & 0.57 & 0.07 \\
3C 273 & $\mathrm{A}$ & $16.93 \pm 3.52$ & $156.64 \pm 34.52$ & 0.94 & 0.00 \\
3C 279 & $\mathrm{B}$ & $4.78 \pm 1.66$ & $41.96 \pm 17.90$ & 0.50 & 0.06 \\
& $\mathrm{C}$ & $1.96 \pm 1.45$ & $12.02 \pm 16.01$ & 0.55 & 0.05 \\
\hline
\end{tabular}

MacDonald N. R., Marscher A. P., Jorstad S. G., Joshi M., 2015, ApJ, 804, 111

Mannheim K., 1993, A\&A, 269, 67

Mao P., Urry C. M., Massaro F., Paggi A., Cauteruccio J., Künzel S. R., 2016, ApJS, 224, 26

Maraschi L., Ghisellini G., Tanzi E. G., Treves A., 1986, ApJ, 310, 325

Marscher A. P., Gear W. K., 1985, ApJ, 298, 114

Mastichiadis A., Kirk J. G., 2002, Publ. Astron. Soc. Australia, 19, 138

Mattox J. R., et al., 1996, ApJ, 461, 396 


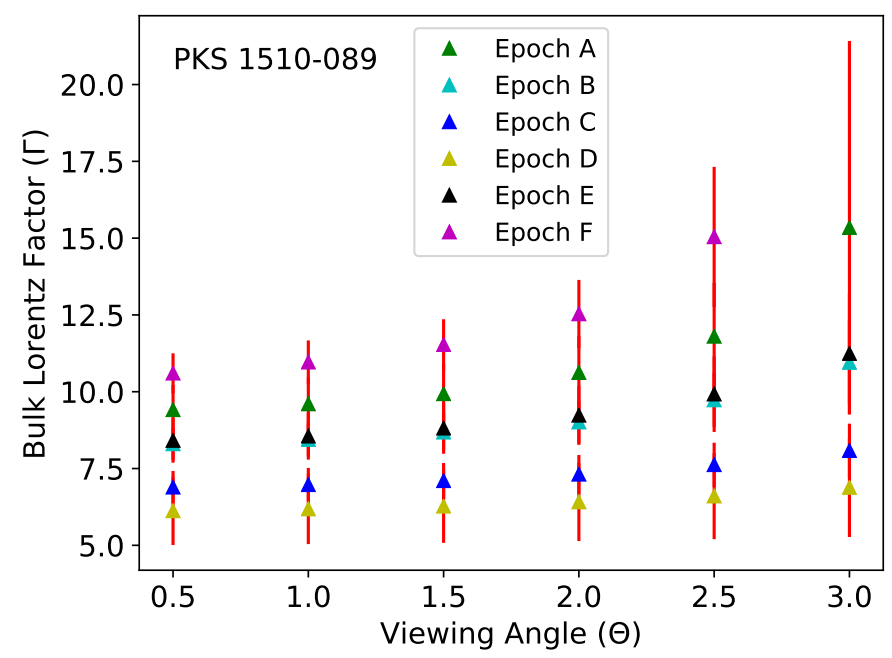

Figure 19. Bulk Lorentz factor v/s viewing angle for the sources PKS 1510-089.

Moore R. L., Stockman H. S., 1981, ApJ, 243, 60

Mücke A., Protheroe R. J., 2001, Astroparticle Physics, 15, 121

Mücke A., Protheroe R. J., Engel R., Rachen J. P., Stanev T., 2003, Astroparticle Physics, 18, 593

Nalewajko K., 2013, MNRAS, 430, 1324

Nalewajko K., Sikora M., Madejski G. M., Exter K., Szostek A., Szczerba R., Kidger M. R., Lorente R., 2012, ApJ, 760, 69

Nolan P. L., et al., 2012, ApJS, 199, 31

Oke J. B., 1967, ApJ, 147, 901

Osterman Meyer A., Miller H. R., Marshall K., Ryle W. T., Aller H., Aller M., Balonek T., 2009, AJ, 138, 1902

Paliya V. S., Sahayanathan S., Stalin C. S., 2015, ApJ, 803, 15

Paliya V. S., Diltz C., Böttcher M., Stalin C. S., Buckley D., 2016, ApJ, 817, 61

Paliya V. S., Zhang H., Böttcher M., Ajello M., Domínguez A., Joshi M., Hartmann D., Stalin C. S., 2018, ApJ, 863, 98

Paliya V. S., Böttcher M., María Del Olmo García A., Domínguez A., Gil de Paz A., Franckowiak A., Garrappa S., Stein R., 2020, arXiv e-prints, p. arXiv:2003.06012

Papadakis I. E., Villata M., Raiteri C. M., 2007, A\&A, 470, 857

Pasierb M., et al., 2020, MNRAS, 492, 1295

Pauliny-Toth I. I. K., Kellermann K. I., 1966, ApJ, 146, 634

Petropoulou M., Nalewajko K., Hayashida M., Mastichiadis A., 2017, MNRAS, 467, L16

Poutanen J., Stern B., 2010, ApJ, 717, L118

Prince R., Majumdar P., Gupta N., 2017, ApJ, 844, 62

Prince R., Gupta N., Nalewajko K., 2019, ApJ, 883, 137

Qian S. J., Britzen S., Krichbaum T. P., Witzel A., 2019, A\&A, 621, A11

Raiteri C. M., et al., 2003, A\&A, 402, 151

Rajput B., Stalin C. S., Sahayanathan S., Rakshit S., Mandal A. K., 2019, MNRAS, 486, 1781

Rajput B., Stalin C. S., Rakshit S., 2020, A\&A, 634, A80

Rakshit S., Stalin C. S., Muneer S., Neha S., Paliya V. S., 2017, ApJ, 835, 275

Rani B., et al., 2018, ApJ, 858, 80

Sahakyan N., 2020, A\&A, 635, A25

Sahayanathan S., Sinha A., Misra R., 2018, Research in Astronomy and Astrophysics, 18, 035

Sarkar A., et al., 2019, ApJ, 887, 185

Schmidt M., 1963, Nature, 197, 1040

Schmidt M., 1965, ApJ, 141, 1295
Shah Z., Jithesh V., Sahayanathan S., Misra R., Iqbal N., 2019, MNRAS, 484, 3168

Shakura N. I., Sunyaev R. A., 1973, A\&A, 24, 337

Shukla A., et al., 2018, ApJ, 854, L26

Sikora M., Begelman M. C., Rees M. J., 1994, ApJ, 421, 153

Singal J., Ko A., Petrosian V., 2014, ApJ, 786, 109

Smith P. S., Montiel E., Rightley S., Turner J., Schmidt G. D., Jannuzi B. T., 2009, preprint, (arXiv:0912.3621)

Stalin C. S., et al., 2009, MNRAS, 399, 1357

Swanenburg B. N., et al., 1978, Nature, 275, 298

Tanner A. M., Bechtold J., Walker C. E., Black J. H., Cutri R. M., 1996, AJ, 112,62

The Fermi-LAT collaboration 2019, arXiv e-prints, p. arXiv:1905.10771

Ulrich M.-H., Maraschi L., Urry C. M., 1997, ARA\&A, 35, 445

Urry C. M., Padovani P., 1995, PASP, 107, 803

Vercellone S., et al., 2011, ApJ, 736, L38

Villata M., et al., 2004, A\&A, 421, 103

Wagner S. J., Witzel A., 1995, ARA\&A, 33, 163

Weisskopf M. C., et al., 2016, The Imaging X-ray Polarimetry Explorer (IXPE). p. 990517, doi:10.1117/12.2235240

Wierzcholska A., Zacharias M., Jankowsky F., Wagner S., H. E. S. S. Collaboration 2019, Galaxies, 7, 21

Wood M., Caputo R., Charles E., Di Mauro M., Magill J., Perkins J. S., FermiLAT Collaboration 2017, in 35th International Cosmic Ray Conference (ICRC2017). p. 824 (arXiv: 1707.09551)

Wu J., Zhou X., Peng B., Ma J., Jiang Z., Chen J., 2005, MNRAS, 361, 155

Xiong D., Bai J., Zhang H., Fan J., Gu M., Yi T., Zhang X., 2017, ApJS, 229, 21

Zhang H., Böttcher M., 2013, ApJ, 774, 18

Zheng Y. G., Zhang L., Huang B. R., Kang S. J., 2013, MNRAS, 431, 2356

This paper has been typeset from a $\mathrm{T}_{\mathrm{E}} \mathrm{X} / \mathrm{LT} \mathrm{E} \mathrm{X}$ file prepared by the author. 